\title{
Lower semicontinuity for an integral functional in $\mathrm{BV}^{*}$
}

\author{
Jan Kristensen and Panu Lahti \\ Mathematical Institute, University of Oxford, \\ Andrew Wiles Building, \\ Radcliffe Observatory Quarter, Woodstock Road, \\ Oxford, OX2 6GG. \\ E-mail: jan.kristensen@maths.ox.ac.uk, \\ lahti@maths.ox.ac.uk
}

July 17,2018

\begin{abstract}
We prove a lower semicontinuity result for a functional of linear growth initially defined by

$$
\int_{\Omega} F\left(\frac{d D u}{d \mu}\right) d \mu
$$

for $u \in \operatorname{BV}\left(\Omega ; \mathbb{R}^{N}\right)$ with $D u \ll \mu$. The positive Radon measure $\mu$ is only assumed to satisfy $\mathcal{L}^{n} \ll \mu$.

Acknowledgments: This research was done while P.L. was visiting the Oxford Centre for Nonlinear Partial Differential Equations (OxPDE) from September 2014 to July 2016. During this time, P.L. was supported by Aalto University as well as the Finnish Cultural Foundation.
\end{abstract}

${ }^{*} 2010$ Mathematics Subject Classification: 49J45, 26B30, 52A99. 


\section{Introduction}

In this work we prove a lower semicontinuity result for a functional of linear growth initially defined in an open set $\Omega \subset \mathbb{R}^{n}$ by

$$
\int_{\Omega} F\left(\frac{d D u}{d \mu}\right) d \mu
$$

for $u \in \mathrm{BV}\left(\Omega ; \mathbb{R}^{N}\right)$ with $D u \ll \mu$. The measure $\mu$ is merely assumed to be a positive finite Radon measure that satisfies $\mathcal{L}^{n} \ll \mu$, where $\mathcal{L}^{n}$ is the Lebesgue measure. For the integrand $F$ we need somewhat stronger assumptions, described in detail below. We refer the reader to Section 2 for precise notation and terminology.

With the choice $\mu=\mathcal{L}^{n}$, this type of result was derived in [4] (see also [5. Section 5.5]), and later in [10] for integrands depending also on $x$ and $u$. The problem was studied without a nonnegativity assumption on $F$ in [15]. These results relied mostly on blow-up techniques. The result in [15] was generalized to $x$-dependent integrands in [14, Theorem 10], relying on the theory of generalized Young measures, which were first introduced by DiPerna and Majda in [8]. With a general measure $\mu$, the problem was studied in the case $p>1$ in [3], and also in [13]. In a more general setting of a metric measure space, the problem was studied in [11].

We first show that the functional (1.1), defined for general $u \in \operatorname{BV}\left(\Omega ; \mathbb{R}^{N}\right)$ by relaxation, has an integral representation

$$
\int_{\Omega} F\left(\frac{d D u}{d \mu}\right) d \mu+\int_{\Omega} F^{\infty}\left(\frac{d D^{s, \mu} u}{d\left|D^{s, \mu} u\right|}\right) d\left|D^{s, \mu} u\right|,
$$

where $D^{s, \mu} u$ is the singular part of $D u$ with respect to $\mu$. Here we require the integrand $F: \mathbb{R}^{N \times n} \rightarrow \mathbb{R}$ be nonnegative and quasiconvex, with linear growth $m|A| \leq F(A) \leq M(1+|A|)$ for some $0<m \leq M$ and all $A \in \mathbb{R}^{N \times n}$, and with a continuous recession function $F^{\infty}$.

Our proof will rely heavily on the theory of generalized Young measures, particularly results derived in [14]. Once we have the above integral representation, we can derive Jensen's inequalities for generalized Young measures with respect to $\mu$, as was done in [14, Theorem 9] with respect to the Lebesgue measure. By using these inequalities, we can then prove the following lower semicontinuity theorem (Theorem 4.4) which is the main result of this work:

Theorem 1.1. Let $\Omega \subset \mathbb{R}^{n}$ be a bounded Lipschitz domain with inner boundary normal $\nu_{\Omega}$, let $\mu$ be a positive finite Radon measure on $\Omega$ with $\mathcal{L}^{n} \ll \mu$, and let $F: \bar{\Omega} \times \mathbb{R}^{N \times n} \rightarrow \mathbb{R}$ be a $\mu \times \mathcal{B}\left(\mathbb{R}^{N \times n}\right)$-measurable integrand with linear 
growth $0 \leq F(x, A) \leq M(1+|A|)$ for some $M \geq 0$, a continuous recession function $F^{\infty}$, and such that $A \mapsto F(x, A)$ is quasiconvex for each fixed $x \in \bar{\Omega}$. Then the functional

$$
\begin{aligned}
\mathcal{F}(u):= & \int_{\Omega} F\left(x, \frac{d D u}{d \mu}\right) d \mu+\int_{\Omega} F^{\infty}\left(x, \frac{d D^{s, \mu} u}{d\left|D^{s, \mu} u\right|}\right) d\left|D^{s, \mu} u\right| \\
& +\int_{\partial \Omega} F^{\infty}\left(x, \frac{u}{|u|} \otimes \nu_{\Omega}\right)|u| d \mathcal{H}^{n-1}
\end{aligned}
$$

is weakly* sequentially lower semicontinuous in $\mathrm{BV}\left(\Omega ; \mathbb{R}^{N}\right)$.

We remark that an easier proof is possible when the Radon-Nikodym derivative of $\mu$ with respect to Lebesgue measure is bounded, and hence that the main contribution is the proof covering the general case. This proof seems to require the assumption about existence of a continuous recession function for the integrand $F$.

\section{Preliminaries}

\section{$2.1 \quad$ Notation}

For $N, n \in \mathbb{N}$, the matrix space $\mathbb{R}^{N \times n}$ will always be equipped with the Euclidean norm $|A|:=\left(\sum_{i=1}^{N} \sum_{j=1}^{n} A_{j}^{i}\right)^{1 / 2}$, where $i$ and $j$ are the row and column indices, respectively. We denote by $B(x, r)$ the open ball in $\mathbb{R}^{n}$ with center $x$ and radius $r$. We denote by $\mathbb{B}^{n}$ the open unit ball in $\mathbb{R}^{n}$ and by $\partial \mathbb{B}^{n}$ the unit sphere. For $a \in \mathbb{R}^{N}$ and $b \in \mathbb{R}^{n}$, we can define the tensor product $a \otimes b=a b^{T} \in \mathbb{R}^{N \times n}$.

We denote the $n$-dimensional Lebesgue measure by $\mathcal{L}^{n}$ and the $s$-dimensional Hausdorff measure by $\mathcal{H}^{s}$. Given any measure $\nu$, the restriction of $\nu$ to a set $A$ is denoted by $\nu \mathrm{L} A$, that is, $\nu \mathrm{L} A(B)=\nu(A \cap B)$. The Borel $\sigma$-algebra on a set $E \subset \mathbb{R}^{n}$ is denoted by $\mathcal{B}(E)$. For open sets $\Omega, \Omega^{\prime} \subset \mathbb{R}^{n}$, by $\Omega \Subset \Omega^{\prime}$ we mean that $\bar{\Omega} \subset \Omega^{\prime}$ and that $\bar{\Omega}$ is compact. We denote by $\mathbb{1}_{E}$ the characteristic function of a set $E$.

If $X$ is a locally compact separable metric space (usually an open or closed subset of $\left.\mathbb{R}^{n}\right)$, let $C_{c}\left(X ; \mathbb{R}^{l}\right)$ be the space of continuous $\mathbb{R}^{l}$-valued functions with compact support in $X$ and let $C_{0}\left(X ; \mathbb{R}^{l}\right)$ be its completion with respect to the $\|\cdot\|_{\infty}$-norm, $l \in \mathbb{N}$. We denote by $\mathcal{M}\left(X ; \mathbb{R}^{l}\right)$ the Banach space of vector-valued finite Radon measures, equipped with the total variation norm $|\mu|(X)<\infty$. By the Riesz representation theorem, $\mathcal{M}\left(X ; \mathbb{R}^{l}\right)$ can be identified with the dual space of $C_{0}\left(X ; \mathbb{R}^{l}\right)$ through the duality pairing 
$\langle\phi, \mu\rangle:=\int_{X} \phi \cdot d \mu:=\sum_{i=1}^{l} \int_{X} \phi_{i} d \mu_{i}$. Thus weak* convergence $\mu_{j} \stackrel{*}{\rightarrow} \mu$ in $\mathcal{M}(X)$ means $\left\langle\phi, \mu_{j}\right\rangle \rightarrow\langle\phi, \mu\rangle$ for all $\phi \in C_{0}\left(X ; \mathbb{R}^{l}\right)$. We denote the set of positive measures and probability measures by $\mathcal{M}^{+}(X)$ and $\mathcal{M}^{1}(X)$, respectively.

For a vector-valued Radon measure $\gamma \in \mathcal{M}\left(X ; \mathbb{R}^{l}\right)$ and a positive Radon measure $\mu \in \mathcal{M}^{+}(X)$, we can write the Radon-Nikodym decomposition $\gamma=$ $\gamma^{a}+\gamma^{s}=\frac{d \gamma}{d \mu} \mu+\gamma^{s}$ of $\gamma$ with respect to $\mu$, where $\frac{d \gamma}{d \mu} \in L^{1}\left(X, \mu ; \mathbb{R}^{l}\right)$.

We write

$$
f_{\Omega} f d \mu:=\frac{1}{\mu(\Omega)} \int_{\Omega} f d \mu
$$

for integral averages (whenever they are defined).

For sets $E \subset \mathbb{R}^{n}, F \subset \mathbb{R}^{l}$ open or closed, a parametrized measure $\left(\nu_{x}\right)_{x \in E} \subset \mathcal{M}(F)$ is a mapping from $E$ to the set $\mathcal{M}(F)$ of Radon measures on $F$. It is said to be weakly* $\mu$-measurable, for $\mu \in \mathcal{M}^{+}(E)$, if $x \mapsto \nu_{x}(B)$ is $\mu$-measurable for all Borel sets $B \in \mathcal{B}(F)$ (it suffices to check this for all relatively open sets). Equivalently, $\left(\nu_{x}\right)_{x \in E}$ is weakly* $\mu$-measurable if the function $x \mapsto \int_{F} f(x, y) d \nu_{x}(y)$ is $\mu$-measurable for every bounded Borel function $f: E \times F \rightarrow \mathbb{R}$ (see [5, Proposition 2.26]). We denote by $L_{w *}^{\infty}(E, \mu ; \mathcal{M}(F)$ ) the set of all weakly* $\mu$-measurable parametrized measures $\left(\nu_{x}\right)_{x \in E} \subset \mathcal{M}(F)$ with the property that $\operatorname{ess}^{\sup _{x \in E}}\left|\nu_{x}\right|(F)<\infty$ (the essential supremum with respect to $\mu$ ). We omit $\mu$ in the notation if $\mu=\mathcal{L}^{n}$.

\subsection{Functions of bounded variation}

The theory of BV functions presented in this section can be found in e.g. the monographs [5, 9, 19], and we will give specific references only for a few key results. Let $\Omega \subset \mathbb{R}^{n}$ be an open set. A function $u \in L^{1}\left(\Omega ; \mathbb{R}^{N}\right)$ is a function of bounded variation, denoted by $u \in \mathrm{BV}\left(\Omega ; \mathbb{R}^{N}\right)$, if its distributional derivative is a bounded $\mathbb{R}^{N \times n}$-valued Radon measure. This means that there exists a (unique) measure $D u \in \mathcal{M}\left(\mathbb{R}^{n} ; \mathbb{R}^{N \times n}\right)$ such that for all $\psi \in C_{c}^{1}(\Omega)$, the integration-by-parts formula

$$
\int_{\Omega} \frac{\partial \psi}{\partial x_{j}} u^{i} d \mathcal{L}^{n}=-\int_{\Omega} \psi d D u_{j}^{i}, \quad i=1 \ldots N, \quad j=1, \ldots, n
$$

holds. We write the Radon-Nikodym decomposition of the variation measure as $D u=\nabla u \mathcal{L}^{n}\left\llcorner\Omega+D^{s} u\right.$.

The space $\operatorname{BV}\left(\Omega ; \mathbb{R}^{N}\right)$ is a Banach space endowed with the norm

$$
\|u\|_{\mathrm{BV}\left(\Omega ; \mathbb{R}^{N}\right)}:=\|u\|_{L^{1}\left(\Omega ; \mathbb{R}^{N}\right)}+|D u|(\Omega) .
$$


Furthermore, we say that a sequence $\left(u_{j}\right) \subset \mathrm{BV}\left(\Omega ; \mathbb{R}^{N}\right)$ converges weakly* to $u \in \operatorname{BV}\left(\Omega ; \mathbb{R}^{N}\right)$ if $u_{j} \rightarrow u$ strongly in $L^{1}\left(\Omega ; \mathbb{R}^{N}\right)$ and $D u_{j} \stackrel{*}{\rightarrow} D u$ in $\mathcal{M}\left(\Omega, \mathbb{R}^{N \times n}\right)$. A norm-bounded sequence in $\operatorname{BV}\left(\Omega ; \mathbb{R}^{N}\right)$, i.e.

$$
\sup _{j \in \mathbb{N}}\left(\|u\|_{L^{1}\left(\Omega ; \mathbb{R}^{N}\right)}+\left|D u_{j}\right|(\Omega)\right)<\infty
$$

always has a weakly* converging subsequence. Conversely, a weakly* converging sequence is norm-bounded in $\operatorname{BV}\left(\Omega, \mathbb{R}^{N}\right)$, see [5, Proposition 3.13]. If $u_{j} \rightarrow u$ in $L^{1}\left(\Omega ; \mathbb{R}^{N}\right)$ and $\left|D u_{j}\right|(\Omega) \rightarrow|D u|(\Omega)$, we say that the $u_{j}$ converge to $u$ strictly. If even

$$
\left\langle D u_{j}\right\rangle(\Omega) \rightarrow\langle D u\rangle(\Omega),
$$

where for a measure $\nu \in \mathcal{M}\left(\mathbb{R}^{n} ; \mathbb{R}^{N \times n}\right)$ with Radon-Nikodym decomposition $\nu=a \mathcal{L}^{n}+\mu^{s}$, we define the measure (related to the minimal surface functional)

$$
\langle\nu\rangle(A):=\int_{A} \sqrt{1+|a|^{2}} d \mathcal{L}^{n}+\left|\mu^{s}\right|(A), \quad A \in \mathcal{B}\left(\mathbb{R}^{n}\right),
$$

then we speak of $\langle\cdot\rangle$-strict convergence. This notion is stronger than strict convergence (this follows e.g. from Theorem 2.2 below), and one can show that it implies that $\left\langle D u_{j}\right\rangle \stackrel{*}{\rightarrow}\langle D u\rangle$ as measures.

For any bounded open set $\Omega \subset \mathbb{R}^{n}$ and $v \in \mathrm{BV}\left(\Omega ; \mathbb{R}^{N}\right)$, we can define the Dirichlet class

$$
\mathrm{BV}_{v}\left(\Omega ; \mathbb{R}^{N}\right):=\left\{u \in \mathrm{BV}\left(\Omega ; \mathbb{R}^{N}\right): w \in \mathrm{BV}\left(\mathbb{R}^{n} ; \mathbb{R}^{N}\right) \text { and }|D w|(\partial \Omega)=0\right\},
$$

where

$$
w:= \begin{cases}u-v & \text { in } \Omega, \\ 0 & \text { in } \mathbb{R}^{n} \backslash \Omega .\end{cases}
$$

The following lemma is proved in e.g. [14, Lemma 1].

Lemma 2.1. Let $\Omega \subset \mathbb{R}^{n}$ be a bounded open set, and let $u \in \operatorname{BV}\left(\Omega ; \mathbb{R}^{N}\right)$. Then there exists $\left(v_{j}\right) \subset \operatorname{BV}_{u}\left(\Omega ; \mathbb{R}^{N}\right) \cap C^{\infty}\left(\Omega ; \mathbb{R}^{N}\right)$ such that $v_{j} \rightarrow u\langle\cdot\rangle$ strictly in $\Omega$.

\subsection{Generalized Young measures}

Most of the theory of generalized Young measures presented in this section is derived in [14].

The symbol $\Omega$ will always denote a bounded open set in $\mathbb{R}^{n}$. We will need the following linear transformations mapping $C\left(\Omega \times \mathbb{R}^{l}\right)$ to $C\left(\Omega \times \mathbb{B}^{l}\right)$ 
and back, where $\mathbb{B}^{l}$ was the open unit ball in $\mathbb{R}^{l}$ : for $f \in C\left(\Omega \times \mathbb{R}^{l}\right)$ and $g \in C\left(\Omega \times \mathbb{B}^{l}\right)$, define

$$
\begin{gathered}
(T f)(x, \hat{A}):=(1-|\hat{A}|) f\left(x, \frac{\hat{A}}{1-|\hat{A}|}\right), \quad x \in \Omega, \quad \hat{A} \in \mathbb{B}^{l}, \quad \text { and } \\
\left(T^{-1} g\right)(x, A):=(1+|A|) g\left(x, \frac{A}{1+|A|}\right), \quad x \in \Omega, \quad A \in \mathbb{R}^{l} .
\end{gathered}
$$

It is an easy calculation to verify that $T^{-1} T f=f$ and $T T^{-1} g=g$. We consider the property

$$
T f \text { extends to a bounded continuous function on } \overline{\Omega \times \mathbb{B}^{l}} \text {. }
$$

In particular, this entails that $f$ has linear growth at infinity, that is, there exists a constant $M \geq 0$ (in fact, $M=\|T f\|_{L^{\infty}\left(\overline{\Omega \times \mathbb{B}^{l}}\right)}$ will do) such that

$$
|f(x, A)| \leq M(1+|A|) \quad \text { for all } x \in \bar{\Omega}, A \in \mathbb{R}^{l} .
$$

We collect all such integrands into the set

$$
\mathbf{E}\left(\Omega ; \mathbb{R}^{l}\right):=\left\{f \in C\left(\Omega \times \mathbb{R}^{l}\right): f \text { satisfies }(2.1)\right\} .
$$

For $f \in \mathbf{E}\left(\Omega ; \mathbb{R}^{l}\right)$, the recession function $f^{\infty}: \bar{\Omega} \times \mathbb{R}^{l} \mapsto \mathbb{R}$ is defined by

$$
f^{\infty}(x, A):=\lim _{\substack{x^{\prime} \rightarrow x \\ A^{\prime} \rightarrow A \\ t \rightarrow \infty}} \frac{f\left(x^{\prime}, t A^{\prime}\right)}{t}, \quad x \in \bar{\Omega}, A \in \mathbb{R}^{l} .
$$

The limit exists since it agrees with $T f$ on $\bar{\Omega} \times \partial \mathbb{B}^{N \times n}$, as can be seen by substituting $t=s /(1-s), s \in(0,1)$, and letting $s \rightarrow 1$. The recession function is clearly positively 1 -homogenous in $A$, that is, $f^{\infty}(x, s A)=s f^{\infty}(x, A)$ for all $s \geq 0$, and thus takes finite values.

We also consider a second class of integrands that is larger than $\mathbf{E}\left(\Omega ; \mathbb{R}^{l}\right)$ and (partially) dispenses with continuity in the $x$-variable. A Carathéodory function is an $\mathcal{L}^{n} \times \mathcal{B}\left(\mathbb{R}^{l}\right)$-measurable function $f: \bar{\Omega} \times \mathbb{R}^{l} \rightarrow \mathbb{R}$ such that $A \mapsto f(x, A)$ is continuous for almost every $x \in \bar{\Omega}$. In fact, it can be shown that it suffices to check measurability of $x \mapsto f(x, A)$ for all fixed $A \in \mathbb{R}^{l}$ (see for example [5, Proposition 5.6]). With this notion, the representation integrands are defined as follows:

$$
\begin{array}{r}
\mathbf{R}\left(\Omega ; \mathbb{R}^{l}\right):=\left\{f: \bar{\Omega} \times \mathbb{R}^{l} \rightarrow \mathbb{R}: \quad\right. \text { Carathéodory with linear growth } \\
\text { at infinity and } \left.\exists f^{\infty} \in C\left(\bar{\Omega} \times \mathbb{R}^{l}\right)\right\} .
\end{array}
$$


A function $f: \mathbb{R}^{N \times n} \rightarrow \mathbb{R}$ is said to be quasiconvex, which we denote by $f \in \mathbf{Q}\left(\mathbb{R}^{N \times n}\right)$, if $f$ is Borel measurable, locally bounded from below, and for some bounded Lipschitz domain $\omega \subset \mathbb{R}^{n}$ and every $A \in \mathbb{R}^{N \times n}$ it holds that

$$
|\omega| f(A) \leq \int_{\omega} f(A+\nabla \psi(x)) d \mathcal{L}^{n}(x) \quad \text { for all } \psi \in W_{0}^{1, \infty}\left(\omega ; \mathbb{R}^{N}\right) .
$$

This definition does not depend on the particular choice of the Lipschitz domain $\omega$ (by an exhaustion argument) and it can be shown that quasiconvex functions are rank one convex, meaning that they are convex along rank one lines (see for example [5, Proposition 5.41]). See [7] for more on quasiconvexity.

A quasiconvex function does not necessarily have a recession function $f^{\infty}$ in the sense of (2.2) (see [16, Theorem 2] for a counterexample), and the notion can be relaxed in the following way: for $f: \mathbb{R}^{N \times n} \rightarrow \mathbb{R}$ the generalized recession function $f^{\#}: \mathbb{R}^{N \times n} \rightarrow \mathbb{R} \cup\{ \pm \infty\}$ is defined by

$$
f^{\#}(A):=\limsup _{\substack{A^{\prime} \rightarrow A \\ t \rightarrow \infty}} \frac{f\left(t A^{\prime}\right)}{t}, \quad A \in \mathbb{R}^{N \times n} .
$$

Quasiconvex functions are globally Lipschitz continuous (see for example [6, Lemma 2.2]) and hence for quasiconvex $f$

$$
f^{\#}(A)=\limsup _{t \rightarrow \infty} \frac{f(t A)}{t}, \quad A \in \mathbb{R}^{N \times n} .
$$

By rank one convexity, the above holds as a limit for all matrices $A$ of rank one.

We have the following version of Reshetnyak's Continuity Theorem, see the appendix of [15], as well as [17, Theorem 3] or [5, Theorem 2.39] for the original result stated for 1-homogenous functions $f$.

Theorem 2.2. Let $\left(\gamma_{j}\right) \subset \mathcal{M}\left(\bar{\Omega} ; \mathbb{R}^{l}\right), \gamma \in \mathcal{M}\left(\bar{\Omega} ; \mathbb{R}^{l}\right)$ with Radon-Nikodym decompositions

$$
\begin{gathered}
\gamma_{j}=a_{j} \mathcal{L}^{n}\left\llcorner\Omega+\gamma_{j}^{s}, \quad \gamma=a \mathcal{L}^{n}\left\llcorner\Omega+\gamma^{s}\right.\right. \\
\text { If } \gamma_{j} \stackrel{*}{\rightarrow} \gamma \text { in } \mathcal{M}\left(\bar{\Omega} ; \mathbb{R}^{l}\right) \text { and }\left\langle\gamma_{j}\right\rangle(\bar{\Omega}) \rightarrow\langle\gamma\rangle(\bar{\Omega}) \text {, then for } \\
\mathcal{F}(\gamma):=\int_{\bar{\Omega}} f(x, a(x)) d \mathcal{L}^{n}+\int_{\bar{\Omega}} f^{\infty}\left(x, \frac{d \gamma^{s}}{d\left|\gamma^{s}\right|}(x)\right) d\left|\gamma^{s}\right|(x) \\
\text { with } f \in \mathbf{E}\left(\Omega, \mathbb{R}^{l}\right) \text {, we have } \mathcal{F}\left(\gamma_{j}\right) \rightarrow \mathcal{F}(\gamma) \text {. }
\end{gathered}
$$


Let $\mu \in \mathcal{M}^{+}(\bar{\Omega})$, and assume that $\mu(\partial \Omega)=0$.

The set of all generalized Young measures $\mathbf{Y}\left(\Omega, \mu ; \mathbb{R}^{l}\right)$ is defined to be the set of all triples $\left(\nu_{x}, \lambda_{\nu}, \nu_{x}^{\infty}\right)$ such that

$$
\begin{array}{ll}
\left(\nu_{x}\right)_{x} \in L_{w *}^{\infty}\left(\Omega, \mu ; \mathcal{M}^{1}\left(\mathbb{R}^{l}\right)\right), & \lambda_{\nu} \in \mathcal{M}^{+}(\bar{\Omega}), \\
\left(\nu_{x}^{\infty}\right)_{x} \in L_{w *}^{\infty}\left(\bar{\Omega}, \lambda_{\nu} ; \mathcal{M}^{1}\left(\partial \mathbb{B}^{l}\right)\right), & x \mapsto\left\langle|\cdot|, \nu_{x}\right\rangle \in \mathrm{L}^{1}(\Omega, \mu) .
\end{array}
$$

Under the duality pairing

$$
\begin{aligned}
& \langle\langle f, \nu\rangle\rangle:=\int_{\Omega}\left\langle f(x, \cdot), \nu_{x}\right\rangle d \mu(x)+\int_{\bar{\Omega}}\left\langle f^{\infty}(x, \cdot), \nu_{x}^{\infty}\right\rangle d \lambda_{\nu}(x) \\
& =\int_{\Omega} \int_{\mathbb{R}^{N \times n}} f(x, A) d \nu_{x}(A) d \mu(x)+\int_{\bar{\Omega}} \int_{\partial \mathbb{B}^{N \times n}} f^{\infty}(x, A) d \nu_{x}^{\infty}(A) d \lambda_{\nu}(x),
\end{aligned}
$$

where $f \in \mathbf{E}\left(\Omega ; \mathbb{R}^{l}\right)$ and $\nu \in \mathbf{Y}\left(\Omega, \mu ; \mathbb{R}^{l}\right)$, the space of Young measures can be considered a part of the dual space $\mathbf{E}\left(\Omega ; \mathbb{R}^{l}\right)^{*}$. We say that a sequence of Young measures $\left(\nu_{j}\right) \subset \mathbf{Y}\left(\Omega, \mu ; \mathbb{R}^{l}\right)$ converges weakly* to $\nu \in \mathbf{Y}\left(\Omega, \mu ; \mathbb{R}^{l}\right)$ if $\left\langle\left\langle f, \nu_{j}\right\rangle\right\rangle \rightarrow\langle\langle f, \nu\rangle\rangle$ for every $f \in \mathbf{E}\left(\Omega ; \mathbb{R}^{l}\right)$.

To every Radon measure $\gamma \in \mathcal{M}\left(\Omega ; \mathbb{R}^{l}\right)$, with Radon-Nikodym decomposition with respect to $\mu$ written as $\gamma=\frac{d \gamma}{d \mu} \mu+\gamma^{s, \mu}$, we associate an elementary Young measure $\varepsilon_{\gamma} \in \mathbf{Y}\left(\Omega, \mu ; \mathbb{R}^{l}\right)$ by

$$
\left(\varepsilon_{\gamma}\right)_{x}:=\delta_{\frac{d \gamma}{d \mu}(x)}, \quad \lambda_{\varepsilon_{\gamma}}:=\left|\gamma^{s, \mu}\right|, \quad\left(\varepsilon_{\gamma}\right)_{x}^{\infty}:=\delta_{p(x)},
$$

where $p:=\frac{d \gamma^{s, \mu}}{d\left|\gamma^{s, \mu}\right|} \in L^{1}\left(\Omega,\left|\gamma^{s, \mu}\right| ; \partial \mathbb{B}^{l}\right)$.

Crucially, we have the following.

Theorem 2.3. Let $\mu \in \mathcal{M}^{+}(\bar{\Omega})$ with $\mu(\partial \Omega)=0$, and let $\left(\gamma_{j}\right) \subset \mathcal{M}\left(\bar{\Omega} ; \mathbb{R}^{l}\right)$ be a sequence of Radon measures that is bounded in the total variation norm, that is, $\sup _{j \in \mathbb{N}}\left|\gamma_{j}\right|(\bar{\Omega})<\infty$. Then there exists a subsequence (not relabeled) and a generalized Young measure $\left(\nu_{x}, \lambda_{\nu}, \nu_{x}^{\infty}\right)$ with

$$
\begin{array}{ll}
\left(\nu_{x}\right)_{x} \in L_{w *}^{\infty}\left(\Omega, \mu ; \mathcal{M}^{1}\left(\mathbb{R}^{l}\right)\right), & \lambda_{\nu} \in \mathcal{M}^{+}(\bar{\Omega}), \\
\left(\nu_{x}^{\infty}\right)_{x} \in L_{w *}^{\infty}\left(\bar{\Omega}, \lambda_{\nu} ; \mathcal{M}^{1}\left(\partial \mathbb{B}^{l}\right)\right), & x \mapsto\left\langle|\cdot|, \nu_{x}\right\rangle \in \mathrm{L}^{1}(\Omega, \mu),
\end{array}
$$

such that $\left\langle\left\langle f, \varepsilon_{\gamma_{j}}\right\rangle\right\rangle \rightarrow\langle\langle f, \nu\rangle\rangle$, or equivalently

$$
\begin{aligned}
f\left(x, \frac{d \gamma_{j}}{d \mu}(x)\right) \mu+f^{\infty}\left(x, \frac{d \gamma_{j}^{s, \mu}}{d\left|\gamma_{j}^{s, \mu}\right|}(x)\right)\left|\gamma_{j}^{s, \mu}\right| & \\
& \stackrel{*}{\longrightarrow}\left\langle f(x, \cdot), \nu_{x}\right\rangle \mu+\left\langle f^{\infty}(x, \cdot), \nu_{x}^{\infty}\right\rangle \lambda_{\nu} \text { in } \mathcal{M}(\bar{\Omega})
\end{aligned}
$$

for every $f \in \mathbf{E}\left(\Omega ; \mathbb{R}^{N \times n}\right)$. 
Proof. This is proved in the case $\mu=\mathcal{L}^{n} \mathrm{~L} \Omega$ in [14, Lemma 2, Corollary 2 , Theorem 7], but the proofs run through also if we replace the Lebesgue measure by a more general $\mu$.

See also [2, Theorem 2.5] for a proof in the case $\gamma_{j}^{s} \equiv 0$.

Corollary 2.4. In the above theorem, (2.6) holds also for every $\mu \times \mathcal{B}\left(\mathbb{R}^{l}\right)$ measurable $f \in \mathbf{R}\left(\Omega ; \mathbb{R}^{l}\right)$.

In the case $\mu=\mathcal{L}^{n}$, (2.6) also holds for every Carathéodory integrand $f: \bar{\Omega} \times \mathbb{R}^{l} \rightarrow \mathbb{R}$ possessing a recession function $f^{\infty}: \bar{\Omega} \times \mathbb{R}^{l} \rightarrow \mathbb{R}$ in the sense of (2.2) for $(x, A) \in(\bar{\Omega} \backslash N) \times \mathbb{R}^{l}$, and $f^{\infty}$ is jointly continuous in $(\bar{\Omega} \backslash N) \times \mathbb{R}^{l}$, where $N \subset \bar{\Omega}$ is a Borel set with $\left(\mathcal{L}^{n}+\lambda_{\nu}\right)(N)=0$.

Note that a Carathéodory function $f: \Omega \times \mathbb{R}^{N} \rightarrow \mathbb{R}$ is by definition $\mathcal{L}^{n} \times \mathcal{B}\left(\mathbb{R}^{N}\right)$-measurable, but here we need the assumption of $\mu \times \mathcal{B}\left(\mathbb{R}^{N}\right)$ measurability.

Proof. Again, this is proved in the case $\mu=\mathcal{L}^{n} L \Omega$ in [14, Proposition 2], but the proof runs through also in the general case with the assumption of $\mu \times \mathcal{B}\left(\mathbb{R}^{N}\right)$-measurability.

In particular, given $u \in \mathrm{BV}\left(\Omega ; \mathbb{R}^{N}\right)$, we can associate to its derivative $D u \in \mathcal{M}\left(\Omega ; \mathbb{R}^{N \times n}\right)$ the Radon-Nikodym decomposition $D u=\frac{d D u}{d \mu} \mu+D^{s, \mu} u$, and then the elementary Young measure $\varepsilon_{D u} \in \mathbf{Y}\left(\Omega, \mu ; \mathbb{R}^{N \times n}\right)$ with

$$
\left(\varepsilon_{D u}\right)_{x}:=\delta_{\frac{d D u}{d \mu}}, \quad \lambda_{\varepsilon_{D u}}:=\left|D^{s, \mu} u\right|, \quad\left(\varepsilon_{D u}\right)_{x}^{\infty}:=\delta_{p(x)},
$$

where $p:=\frac{D^{s, \mu} u}{\mid D^{s, \mu} u} \in L^{1}\left(\Omega,\left|D^{s, \mu} u\right| ; \partial \mathbb{B}^{N \times n}\right)$.

For a norm-bounded sequence $\left(u_{j}\right) \subset \mathrm{BV}\left(\Omega ; \mathbb{R}^{N}\right)$, we say that the derivatives $D u_{j}$ generate the generalized Young measure

$$
\nu=\left(\nu_{x}, \lambda_{\nu}, \nu_{x}^{\infty}\right) \in \mathbf{Y}\left(\Omega ; \mathbb{R}^{N \times n}\right),
$$

if for all $f \in \mathbf{E}\left(\Omega, \mathbb{R}^{N \times n}\right)$ we have that $\left\langle\left\langle f, \varepsilon_{D u_{j}}\right\rangle\right\rangle \rightarrow\langle\langle f, \nu\rangle\rangle$ for all $f \in$ $\mathbf{E}\left(\Omega ; \mathbb{R}^{N \times n}\right)$, or equivalently

$$
\begin{aligned}
f\left(x, \frac{d D u_{j}}{d \mu}\right) \mu+f^{\infty}\left(x, \frac{d D^{s, \mu} u_{j}}{d\left|D^{s, \mu} u_{j}\right|}(x)\right)\left|D^{s, \mu} u_{j}\right| \\
\quad \stackrel{*}{\rightarrow}\left\langle f(x, \cdot), \nu_{x}\right\rangle \mu+\left\langle f^{\infty}(x, \cdot), \nu_{x}^{\infty}\right\rangle \lambda_{\nu} \quad \text { in } \mathcal{M}(\bar{\Omega}) .
\end{aligned}
$$

We call such a generalized Young measure a gradient Young measure. Since $\left(u_{j}\right)$ is norm-bounded, we have $u_{j} \stackrel{*}{\rightarrow} u$ for some $u \in \operatorname{BV}\left(\Omega ; \mathbb{R}^{N}\right)$. The barycenter of a generalized Young measure $\nu \in \mathbf{Y}\left(\Omega ; \mathbb{R}^{N \times n}\right)$ is defined as the measure

$$
\left\langle\text { id }, \nu_{x}\right\rangle \mu+\left\langle\text { id, } \nu_{x}^{\infty}\right\rangle \lambda_{\nu}
$$


Note that by choosing $f$ to be the identity on $\mathbb{R}^{N \times n}$ in (2.7) (componentwise, to be precise), we obtain that $D u$ is the restriction of the barycenter to $\Omega$.

In the case $\mu=\mathcal{L}^{n}$, we have the following Jensen's inequalities for gradient Young measures, which are part of [14, Theorem 9].

Theorem 2.5. Let $u \in \operatorname{BV}\left(\Omega ; \mathbb{R}^{N}\right)$ and let $\nu \in \mathbf{Y}\left(\Omega ; \mathbb{R}^{N \times n}\right), \nu=\left(\nu_{x}, \lambda_{\nu}, \nu_{x}^{\infty}\right)$ be a gradient Young measure with barycenter Du and satisfying $\lambda(\partial \Omega)=0$. Then the following hold for any quasiconvex $f: \mathbb{R}^{N \times n} \rightarrow \mathbb{R}$ with linear growth (that is, $|F(A)| \leq M(|A|+1)$ for all $A \in \mathbb{R}^{N \times n}$ and some $M \geq 0$ ):

$$
\begin{aligned}
& f(\nabla u(x)) \leq\left\langle f, \nu_{x}\right\rangle+\left\langle f^{\#}, \nu_{x}^{\infty}\right\rangle \frac{d \lambda_{\nu}}{d \mathcal{L}^{n}}(x) \quad \text { for } \mathcal{L}^{n} \text {-almost every } x \in \Omega, \\
& f^{\#}\left(\frac{d D^{s} u}{d\left|D^{s} u\right|}\right)\left|D^{s} u\right| \leq\left\langle f^{\#}, \nu_{x}^{\infty}\right\rangle \lambda_{\nu}^{s} \quad \text { as measures. }
\end{aligned}
$$

\section{The integral representation}

Let $F: \mathbb{R}^{N \times n} \mapsto \mathbb{R}$ be quasiconvex, with linear growth

$$
m|A| \leq F(A) \leq M(1+|A|) \quad \text { for all } A \in \mathbb{R}^{N \times n},
$$

for some $0<m \leq M$, such that the recession function $F^{\infty}$ exists in the sense of (2.2). Let $\Omega \subset \mathbb{R}^{n}$ be a bounded open set with $\mathcal{L}^{n}(\partial \Omega)=0$, and let $\mu \in \mathcal{M}^{+}(\Omega)$ with $\mathcal{L}^{n} \ll \mu$. We define a Sobolev space with respect to $\mu$ by

$$
W_{\mu}^{1,1}\left(\Omega ; \mathbb{R}^{N}\right):=\left\{u \in \operatorname{BV}\left(\Omega ; \mathbb{R}^{N}\right): D u \ll \mu\right\} .
$$

We consider the functional

$$
\begin{aligned}
\mathcal{F}_{*}(u, \Omega):=\inf \left\{\liminf _{j \rightarrow \infty} \int_{\Omega} F\left(\frac{d D u_{j}}{d \mu}\right) d \mu,\right. & u_{j} \in W_{\mu}^{1,1}\left(\Omega ; \mathbb{R}^{N}\right) \\
& \left.u_{j} \rightarrow u \text { in } L^{1}\left(\Omega ; \mathbb{R}^{N}\right)\right\}
\end{aligned}
$$

for $u \in \operatorname{BV}\left(\Omega ; \mathbb{R}^{N}\right)$. Note that the convergence above is in $L^{1}\left(\Omega ; \mathbb{R}^{N}\right)$ with respect to the Lebesgue measure $\mathcal{L}^{n}$, not $\mu$. We will prove an integral representation for the above functional. The representation is

$$
\int_{\Omega} F\left(\frac{d D u}{d \mu}\right) d \mu+\int_{\Omega} F^{\infty}\left(\frac{d D^{s, \mu} u}{d\left|D^{s, \mu} u\right|}\right) d\left|D^{s, \mu} u\right|
$$

for any $u \in \operatorname{BV}\left(\Omega ; \mathbb{R}^{N}\right)$, where $D^{s, \mu} u$ is the singular part of the variation measure $D u$ with respect to $\mu$.

Initially we will work with a more restricted class of integrands, defined as follows. 
Definition 3.1. Define the class $\mathbf{S Q}\left(\mathbb{R}^{N \times n}\right)$ of special quasiconvex integrands as quasiconvex functions $F: \mathbb{R}^{N \times n} \rightarrow \mathbb{R}$ with linear growth $|F(A)| \leq M(1+$ $|A|)$ for some $M \geq 0$, such that for some parameters $i, r_{i}>0, F(A)=$ $F^{\infty}(A)-i$ for $|A| \geq r_{i}$, and $F^{\infty}(A) \geq|A| / i$ for all $A \in \mathbb{R}^{N \times n}$.

Note that the existence of the recession function $F^{\infty}$ in the sense of (2.2) is part of the definition. (We could equally well require above that $F(A)=$ $F^{\#}(A)-i$ for $|A| \geq r_{i}$, recall $(2.4)$, as this would imply the existence of $F^{\infty}$.) Clearly $\mathbf{S Q}\left(\mathbb{R}^{N \times n}\right) \subset \mathbf{E}\left(\Omega ; \mathbb{R}^{N \times n}\right)$ (constant in the $x$-variable).

Given $F \in \mathbf{Q}\left(\mathbb{R}^{N \times n}\right)$ with linear growth $0 \leq F(A) \leq M(1+|A|)$ for some $M \geq 0$, we can define $G_{i}(A):=\max \left\{F(A), F^{\#}(A)+|A| / i-i\right\}$ for each $i \in \mathbb{N}$, and then it is shown in [12, Lemma 6.3] that $G_{i} \in \mathbf{S Q}\left(\mathbb{R}^{N \times n}\right)$ and that $G_{i}(A) \searrow F(A)$ and $\left(G_{i}\right)^{\infty}(A) \searrow F^{\infty}(A)$ for every $A \in \mathbb{R}^{N \times n}$. We will use this fact on a number of occasions.

\subsection{Estimate from below}

In order to obtain the integral representation, we first prove the estimate from below.

Proposition 3.2. Let $\Omega \subset \mathbb{R}^{n}$ be a bounded open set with $\mathcal{L}^{n}(\partial \Omega)=0$, let $\mu \in \mathcal{M}^{+}(\Omega)$ with $\mathcal{L}^{n} \ll \mu$, let $F \in \mathbf{R}\left(\Omega ; \mathbb{R}^{N \times n}\right) \cap \mathbf{Q}\left(\mathbb{R}^{N \times n}\right)$ with

$$
m|A| \leq F(A) \leq M(1+|A|)
$$

for some $0<m \leq M$, and let $u \in \operatorname{BV}\left(\Omega ; \mathbb{R}^{N}\right)$. Then we have

$$
\mathcal{F}_{*}(u, \Omega) \geq \int_{\Omega} F\left(\frac{d D u}{d \mu}\right) d \mu+\int_{\Omega} F^{\infty}\left(\frac{d D^{s, \mu} u}{d\left|D^{s, \mu} u\right|}\right) d\left|D^{s, \mu} u\right|
$$

Write the Radon-Nikodym decomposition of $\mu$ as $\mu=a \mathcal{L}^{n}+\mu^{s}$, with $a \in L^{1}(\Omega)$. We prove the theorem by considering separately the sets where the absolutely continuous part and the singular part of $\mu$ are carried.

\subsubsection{The absolutely continuous part}

The following lemma gives, in essence, the estimate from below for the set where the absolutely continuous part of $\mu$ is carried. At this point, we make the extra assumption that $F$ is a special quasiconvex integrand.

Lemma 3.3. Let $\Omega \subset \mathbb{R}^{n}$ be a bounded open set with $\mathcal{L}^{n}(\partial \Omega)=0$, let $\mu \in \mathcal{M}^{+}(\Omega)$ with $\mathcal{L}^{n} \ll \mu$, and let $F \in \mathbf{S Q}\left(\mathbb{R}^{N \times n}\right)$ with parameters $i, r_{i}>0$, 
and with linear growth $0 \leq F(A) \leq M(1+|A|)$. Then for any open $U \subset \Omega$ and any sequence $\left(u_{j}\right) \subset W_{\mu}^{1,1}\left(\Omega ; \mathbb{R}^{N}\right)$ with $u_{j} \rightarrow u$ in $L^{1}\left(\Omega ; \mathbb{R}^{N}\right)$ and

$$
\liminf _{j \rightarrow \infty} \int_{\Omega} F\left(\frac{d D u_{j}}{d \mu}\right) d \mu<\infty,
$$

we have

$$
\begin{aligned}
\liminf _{j \rightarrow \infty} & \int_{U} F\left(\frac{d D u_{j}}{d \mu}\right) d \mu \\
& \geq \int_{U} F\left(\frac{\nabla u}{a}\right) a d \mathcal{L}^{n}+\int_{U} F^{\infty}\left(\frac{d D^{s} u}{d\left|D^{s} u\right|}\right) d\left|D^{s} u\right|-\left(M r_{i}+i\right) \mu^{s}(U)
\end{aligned}
$$

Proof. Since $\mathcal{L}^{n} \ll \mu$, we can assume that $a>0$ everywhere in $\Omega$. Pick a subsequence of $\left(u_{j}\right)$ (not relabeled) that gives the limit in (3.4). Since $F(A)=F^{\infty}(A)-i \geq|A| / i-i$ for all $|A| \geq r_{i}$, we have by (3.3) that $\left(u_{j}\right)$ is a norm-bounded sequence in $\operatorname{BV}\left(\Omega ; \mathbb{R}^{N}\right)$. Thus $u_{j} \stackrel{*}{\rightarrow} u$ in $\operatorname{BV}\left(\Omega ; \mathbb{R}^{N}\right)$. By Theorem [2.3, the derivatives $D u_{j}$ generate a generalized Young measure $\left(\nu_{x}, \lambda_{\nu}, \nu_{x}^{\infty}\right)$ with respect to the Lebesgue measure, with $\lambda_{\nu} \in \mathcal{M}^{+}(\bar{\Omega})$ and

$$
\left(\nu_{x}\right)_{x} \in L_{w^{*}}^{\infty}\left(\Omega ; \mathcal{M}^{1}\left(\mathbb{R}^{N \times n}\right)\right), \quad\left(\nu_{x}^{\infty}\right)_{x} \in L_{w^{*}}^{\infty}\left(\bar{\Omega}, \lambda_{\nu} ; \mathcal{M}^{1}\left(\partial \mathbb{B}^{N \times n}\right)\right) .
$$

This means that for every representation integrand $f \in \mathbf{R}\left(\Omega ; \mathbb{R}^{N \times n}\right)$ and every integrand satisfying the conditions of the latter part of Corollary 2.4, we have

$$
\begin{aligned}
& f\left(x, \nabla u_{j}(x)\right) \mathcal{L}^{n}\left\llcorner\Omega+f^{\infty}\left(x, \frac{d D^{s} u_{j}}{d\left|D^{s} u_{j}\right|}\right)\left|D^{s} u_{j}\right|\right. \\
& \stackrel{*}{\longrightarrow}\left\langle f(x, \cdot), \nu_{x}\right\rangle \mathcal{L}^{n}\left\llcorner\Omega+\left\langle f^{\infty}(x, \cdot), \nu_{x}^{\infty}\right\rangle \lambda_{\nu} \quad \text { in } \mathcal{M}(\bar{\Omega}) .\right.
\end{aligned}
$$

First assume that $\mathcal{L}^{n}(\partial U)=\lambda_{\nu}(\partial U)=0$. Let us start computing

$$
\begin{aligned}
\int_{U} F\left(\frac{d D u_{j}}{d \mu}\right) d \mu & =\int_{U} F\left(\frac{d D u_{j}}{d\left(a \mathcal{L}^{n}\right)}\right) a d \mathcal{L}^{n}+\int_{U} F\left(\frac{d D u_{j}}{d \mu^{s}}\right) d \mu^{s} \\
& =\int_{U} F\left(\frac{\nabla u_{j}}{a}\right) a d \mathcal{L}^{n}+\int_{U} F\left(\frac{d D^{s} u_{j}}{d \mu^{s}}\right) d \mu^{s} \\
& =: I_{j}+I I_{j} .
\end{aligned}
$$

We wish to analyze the term $I_{j}$ by using the fact that $D u_{j}$ generates a generalized Young measure. However, the function

$$
(x, A) \mapsto F\left(\frac{A}{a(x)}\right) a(x) \mathbb{1}_{U}(x)
$$


does not necessarily satisfy the conditions of the latter part of Corollary 2.4: while it is a Carathéodory function, its recession function need not be continuous as required. To overcome this problem, we define the super-level sets of $a$ :

$$
E_{m}:=\{x \in \Omega: a(x)>m\}, \quad m \in \mathbb{N} .
$$

Recall that $a(x)>0$ for every $x \in \Omega$. Denoting the minimum of $a$ and $m$ by $a \wedge m$, by the fact that $F(A)=F^{\infty}(A)-i$ for all $|A| \geq r_{i}$ we have for any $x \in U$ and $A \in \partial \mathbb{B}^{N \times n}$

$$
\begin{aligned}
& \limsup _{\substack{x^{\prime} \rightarrow x \\
A^{\prime} \rightarrow A \\
t \rightarrow \infty}} \frac{F\left(\frac{t A^{\prime}}{a\left(x^{\prime}\right) \wedge m}\right)}{t} a\left(x^{\prime}\right) \wedge m=\limsup _{\substack{A^{\prime} \rightarrow A \\
t \rightarrow \infty}} \frac{F\left(t A^{\prime}\right)}{t} \\
& \quad=\limsup _{\substack{A^{\prime} \rightarrow A \\
t \rightarrow \infty}} \frac{F^{\infty}\left(t A^{\prime}\right)-i}{t}=\limsup _{\substack{A^{\prime} \rightarrow A \\
t \rightarrow \infty}} \frac{t F^{\infty}\left(A^{\prime}\right)}{t}=\limsup _{A^{\prime} \rightarrow A} F^{\infty}\left(A^{\prime}\right)=F^{\infty}(A)
\end{aligned}
$$

by the (Lipschitz) continuity of $F^{\infty}$. Note that the first equality is not necessarily true unless we take the minimum of $a$ with $m$. Also, we now see that all of the limit superiors above are in fact limits. We conclude that

$$
(x, A) \mapsto F\left(\frac{A}{(a \wedge m)(x)}\right)(a \wedge m)(x) \mathbb{1}_{U}(x)
$$

satisfies the conditions of the latter part of Corollary 2.4. Fix $m \in \mathbb{N}$. By the fact that $F(A)=F^{\infty}(A)-i$ for all $|A| \geq r_{i}$, we can write

$$
\begin{aligned}
I_{j}- & \int_{U} F\left(\frac{\nabla u_{j}}{a \wedge m}\right) a \wedge m d \mathcal{L}^{n}=\int_{U \cap E_{m}} F\left(\frac{\nabla u_{j}}{a}\right) a-F\left(\frac{\nabla u_{j}}{m}\right) m d \mathcal{L}^{n} \\
= & \int_{U \cap E_{m} \cap\left\{\left|\nabla u_{j}\right|<a r_{i}\right\}} F\left(\frac{\nabla u_{j}}{a}\right) a-F\left(\frac{\nabla u_{j}}{m}\right) m d \mathcal{L}^{n} \\
& \quad+\int_{U \cap E_{m} \cap\left\{\left|\nabla u_{j}\right| \geq a r_{i}\right\}} F^{\infty}\left(\frac{\nabla u_{j}}{a}\right) a-F^{\infty}\left(\frac{\nabla u_{j}}{m}\right) m-i(a-m) d \mathcal{L}^{n} \\
= & \int_{U \cap E_{m} \cap\left\{\left|\nabla u_{j}\right|<a r_{i}\right\}} F\left(\frac{\nabla u_{j}}{a}\right) a-F\left(\frac{\nabla u_{j}}{m}\right) m d \mathcal{L}^{n} \\
& \quad-\int_{U \cap E_{m} \cap\left\{\left|\nabla u_{j}\right| \geq a r_{i}\right\}} i(a-m) d \mathcal{L}^{n} \\
:= & \varepsilon_{m} .
\end{aligned}
$$


We have by the linear growth of $F$

$$
\begin{aligned}
\left|\int_{U \cap E_{m} \cap\left\{\left|\nabla u_{j}\right|<a r_{i}\right\}} F\left(\frac{\nabla u_{j}}{a}\right) a-F\left(\frac{\nabla u_{j}}{m}\right) m d \mathcal{L}^{n}\right| \\
\quad \leq \int_{U \cap E_{m} \cap\left\{\left|\nabla u_{j}\right|<a r_{i}\right\}} F\left(\frac{\nabla u_{j}}{a}\right) a+F\left(\frac{\nabla u_{j}}{m}\right) m d \mathcal{L}^{n} \\
\quad \leq \int_{U \cap E_{m}} M\left(1+r_{i}\right) a+M\left(1+a r_{i} / m\right) m d \mu \\
\quad \leq \int_{U \cap E_{m}} 2 M a\left(1+r_{i}\right) d \mu .
\end{aligned}
$$

Clearly this last quantity converges to zero as $m \rightarrow \infty$, as does the second term of $\varepsilon_{m}$, so in total $\varepsilon_{m} \rightarrow 0$ as $m \rightarrow \infty$.

By the fact that the derivatives $D u_{j}$ generate a generalized Young measure (recall (3.5)) and the fact that the integrand (3.7) satisfies the conditions of the latter part of Corollary 2.4 and has recession function $F^{\infty}$ in $U$, we have

$$
\begin{aligned}
I_{j} & +\int_{U} F^{\infty}\left(\frac{d D^{s} u_{j}}{d\left|D^{s} u_{j}\right|}\right) d\left|D^{s} u_{j}\right|-\varepsilon_{m} \\
& =\int_{U} F\left(\frac{\nabla u_{j}}{a \wedge m}\right) a \wedge m d \mathcal{L}^{n}+\int_{U} F^{\infty}\left(\frac{d D^{s} u_{j}}{d\left|D^{s} u_{j}\right|}\right) d\left|D^{s} u_{j}\right| \\
& \rightarrow \int_{U} \int_{\mathbb{R}^{N \times n}} F\left(\frac{A}{a \wedge m}\right) a \wedge m d \nu_{x}(A) d \mathcal{L}^{n}+\int_{U} \int_{\partial \mathbb{B}^{N \times n}} F^{\infty}(A) d \nu_{x}^{\infty}(A) d \lambda_{\nu}
\end{aligned}
$$

as $j \rightarrow \infty$. Recalling (3.6), let us then consider the term $I I_{j}$. Since $F(A)=$ 


$$
\begin{aligned}
& F^{\infty}(A)-i \text { for all }|A| \geq r_{i}, \text { we estimate } \\
& \begin{aligned}
I I_{j} & -\int_{U} F^{\infty}\left(\frac{d D^{s} u_{j}}{d\left|D^{s} u_{j}\right|}\right) d\left|D^{s} u_{j}\right| \\
= & \int_{U} F\left(\frac{d D^{s} u_{j}}{d \mu^{s}}\right) d \mu^{s}-\int_{U} F^{\infty}\left(\frac{d D^{s} u_{j}}{d \mu^{s}}\right) d \mu^{s} \\
= & \int_{U \cap\left\{\left|d D^{s} u_{j} / d \mu^{s}\right|<r_{i}\right\}} F\left(\frac{d D^{s} u_{j}}{d \mu^{s}}\right) d \mu^{s}-i \mu^{s}\left(U \cap\left\{\left|d D^{s} u_{j} / d \mu^{s}\right| \geq r_{i}\right\}\right) \\
& \quad-\int_{U \cap\left\{\left|d D^{s} u_{j} / d \mu^{s}\right|<r_{i}\right\}} F^{\infty}\left(\frac{d D^{s} u_{j}}{d \mu^{s}}\right) d \mu^{s} \quad F^{\infty}\left(\frac{d D^{s} u_{j}}{d \mu^{s}}\right) d \mu^{s} \\
\geq & -i \mu^{s}\left(U \cap\left\{\left|d D^{s} u_{j} / d \mu^{s}\right| \geq r_{i}\right\}\right)-\iint_{U \cap\left\{\left|d D^{s} u_{j} / d \mu^{s}\right|<r_{i}\right\}} M\left|\frac{d D^{s} u_{j}}{d \mu^{s}}\right| d \mu^{s} \\
\geq & -i \mu^{s}(U)-\int_{U \cap\left\{\left|d D^{s} u_{j} / d \mu^{s}\right|<r_{i}\right\}} \quad \\
\geq & -i \mu^{s}(U)-M r_{i} \mu^{s}(U) .
\end{aligned}
\end{aligned}
$$

Combining (3.6), (3.8), and (3.9), we get by Jensen's inequalities for generalized Young measures given in Theorem 2.5,

$$
\begin{aligned}
\liminf _{j \rightarrow \infty} \int_{U} F\left(\frac{d D u_{j}}{d \mu}\right) d \mu=\liminf _{j \rightarrow \infty}\left(I_{j}+I I_{j}\right) \\
\geq \int_{U} \int_{\mathbb{R}^{N \times n}} F\left(\frac{A}{a \wedge m}\right) a \wedge m d \nu_{x}(A) d \mathcal{L}^{n}+\int_{U} \int_{\partial \mathbb{B}^{N \times n}} F^{\infty}(A) d \nu_{x}^{\infty}(A) d \lambda_{\nu} \\
\quad+\varepsilon_{m}-\left(M r_{i}+i\right) \mu^{s}(U) \\
\geq \int_{U} F\left(\frac{\nabla u}{a \wedge m}\right) a \wedge m d \mathcal{L}^{n}+\int_{U} F^{\infty}\left(\frac{d D^{s} u}{d\left|D^{s} u\right|}\right) d\left|D^{s} u\right| \\
\quad+\varepsilon_{m}-\left(M r_{i}+i\right) \mu^{s}(U) \\
\geq \int_{U} F\left(\frac{\nabla u}{a}\right) a \wedge m d \mathcal{L}^{n}+\int_{U} F^{\infty}\left(\frac{d D^{s} u}{d\left|D^{s} u\right|}\right) d\left|D^{s} u\right| \\
\quad+\varepsilon_{m}-\left(M r_{i}+i\right) \mu^{s}(U) \\
\rightarrow \int_{U} F\left(\frac{\nabla u}{a}\right) a d \mathcal{L}^{n}+\int_{U} F^{\infty}\left(\frac{d D^{s} u}{d\left|D^{s} u\right|}\right) d\left|D^{s} u\right|-\left(M r_{i}+i\right) \mu^{s}(U)
\end{aligned}
$$

as $m \rightarrow \infty$, by the monotone convergence theorem. Finally, if $U$ does not satisfy $\lambda_{\nu}(\partial U)=0$ or $\mathcal{L}^{n}(\partial U)=0$, we define

$$
U_{\kappa}:=\left\{x \in U: \operatorname{dist}\left(x, U^{c}\right)>\kappa\right\}, \quad \kappa>0,
$$


and then $\lambda_{\nu}\left(\partial U_{\kappa}\right)=0$ and $\mathcal{L}^{n}\left(\partial U_{\kappa}\right)=0$ for all but at most countably many $\kappa>0$ by the fact that these are finite measures on $U$. For such values of $\kappa$ we write

$$
\begin{aligned}
\liminf _{j \rightarrow \infty} \int_{U} F\left(\frac{d D u_{j}}{d \mu}\right) d \mu \\
\geq \liminf _{j \rightarrow \infty} \int_{U_{\kappa}} F\left(\frac{d D u_{j}}{d \mu}\right) d \mu \\
\geq \int_{U_{\kappa}} F\left(\frac{\nabla u}{a}\right) a d \mathcal{L}^{n}+\int_{U_{\kappa}} F^{\infty}\left(\frac{d D^{s} u}{d\left|D^{s} u\right|}\right) d\left|D^{s} u\right|-\left(M r_{i}+i\right) \mu^{s}\left(U_{\kappa}\right) \\
\quad \rightarrow \int_{U} F\left(\frac{\nabla u}{a}\right) a d \mathcal{L}^{n}+\int_{U} F^{\infty}\left(\frac{d D^{s} u}{d\left|D^{s} u\right|}\right) d\left|D^{s} u\right|-\left(M r_{i}+i\right) \mu^{s}(U)
\end{aligned}
$$

as $\kappa \rightarrow 0$, by the monotone convergence theorem.

\subsubsection{The singular part}

Let us then consider the set where $\mu^{s}$ is carried. We prove the following lemma.

Lemma 3.4. Let $\Omega \subset \mathbb{R}^{n}$ be a bounded open set, let $\mu \in \mathcal{M}^{+}(\Omega)$, and let $F \in \mathbf{S Q}\left(\mathbb{R}^{N \times n}\right)$ with $F \geq 0$. Then for any sequence $\left(u_{j}\right) \subset W_{\mu}^{1,1}\left(\Omega ; \mathbb{R}^{N}\right)$ with $u_{j} \rightarrow u$ in $L^{1}\left(\Omega ; \mathbb{R}^{N}\right)$ and

$$
\liminf _{j \rightarrow \infty} \int_{\Omega} F\left(\frac{d D u_{j}}{d \mu}\right) d \mu<\infty
$$

we have for any ball $B(y, r) \subset \Omega$

$$
\int_{B(y, r)} F\left(\frac{d D^{s} u}{d \mu^{s}}\right) d \mu^{s} \leq \liminf _{j \rightarrow \infty} \int_{B(y, r)} F\left(\frac{d D u_{j}}{d \mu}\right) d \mu .
$$

Proof. Note again that it is enough to prove the result for a subsequence. Let $i, r_{i}>0$ be the parameters of $F$, see Definition 3.1. Since $F(A)=$ $F^{\infty}(A)-i \geq|A| / i-i$ for all $|A| \geq r_{i}$, the sequence $\frac{d D u_{j}}{d \mu}$ is norm-bounded in $L^{1}\left(\Omega, \mu ; \mathbb{R}^{N \times n}\right)$, implying that $\left(u_{j}\right)$ is a norm-bounded sequence in $\operatorname{BV}\left(\Omega ; \mathbb{R}^{N}\right)$. By Theorem 2.3 we know that with respect to $\mu$, a subsequence of $D u_{j}$ (not relabeled) generates a generalized Young measure $\left(\nu_{x}, \lambda_{\nu}, \nu_{x}^{\infty}\right)$, with $\lambda_{\nu} \in \mathcal{M}^{+}(\bar{\Omega})$ and

$$
\left(\nu_{x}\right)_{x} \in L_{w^{*}}^{\infty}\left(\Omega, \mu ; \mathcal{M}^{1}\left(\mathbb{R}^{N \times n}\right)\right), \quad\left(\nu_{x}^{\infty}\right)_{x} \in L_{w^{*}}^{\infty}\left(\bar{\Omega}, \mu ; \mathcal{M}^{1}\left(\partial \mathbb{B}^{N \times n}\right)\right) .
$$


This means in particular that for every integrand $f \in \mathbf{E}\left(\Omega ; \mathbb{R}^{N \times n}\right)$,

$$
\begin{aligned}
f\left(\frac{d D u_{j}}{d \mu}\right) \mu & \stackrel{*}{\rightarrow}\left\langle f, \nu_{x}\right\rangle \mu+\left\langle f^{\infty}, \nu_{x}^{\infty}\right\rangle \lambda_{\nu} \\
& =\left(\left\langle f, \nu_{x}\right\rangle a+\left\langle f^{\infty}, \nu_{x}^{\infty}\right\rangle \frac{d \lambda_{\nu}}{d \mathcal{L}^{n}}\right) \mathcal{L}^{n}+\left\langle f, \nu_{x}\right\rangle \mu^{s}+\left\langle f^{\infty}, \nu_{x}^{\infty}\right\rangle \lambda_{\nu}^{s}
\end{aligned}
$$

in $\mathcal{M}(\Omega)$. By Alberti's rank one theorem, see [1], we have $\mu^{s}$-almost everywhere that

$$
\frac{d D u_{j}}{d \mu^{s}}=\xi_{j} \otimes \eta, \quad \frac{d D u}{d \mu^{s}}=\xi \otimes \eta,
$$

where $\xi_{j}, \xi \in \mathbb{R}^{N}$ and $\eta \in \partial \mathbb{B}^{n}$. Note that $\eta$ does not depend on $j$. We show that for $\mu^{s}$-almost every $x \in \Omega$, the measure $\nu_{x}$ is carried on the hyperplane $\mathbb{R}^{N} \otimes \eta(x)$. For this, fix $\varepsilon>0$ and fix a point $x_{0} \in \Omega$. Excluding a $\mu^{s}$ negligible set, we can assume by the Besicovitch differentiation theorem (see e.g. [5, Theorem 2.22]) that for some radius $r>0$, we have $B\left(x_{0}, r\right) \subset \Omega$ and

$$
f_{B\left(x_{0}, r\right)}\left|\eta-\eta\left(x_{0}\right)\right| d \mu^{s}<\varepsilon \quad \text { and } \quad \int_{B\left(x_{0}, r\right)} a d \mathcal{L}^{n}<\varepsilon \mu\left(B\left(x_{0}, r\right)\right) .
$$

Fix $R \geq 1$ and define

$$
f(A):=\min \left\{1, \operatorname{dist}\left(A,\left(\mathbb{R}^{N} \otimes \eta\left(x_{0}\right)\right) \cup B(0, R)^{c}\right)\right\} ;
$$

note that there is no $x$-dependence, and $f \in \mathbf{E}\left(\Omega ; \mathbb{R}^{N \times n}\right)$. Since $f(\xi \otimes \eta)=0$ for $|\xi| \geq R$ and $|\eta|=1$ and since $f$ is 1-Lipschitz,

$$
\begin{gathered}
\left|f_{B\left(x_{0}, r\right)} f\left(\xi_{j} \otimes \eta\right) d \mu^{s}-f_{B\left(x_{0}, r\right)} f\left(\xi_{j} \otimes \eta\left(x_{0}\right)\right) d \mu^{s}\right| \\
\leq R f_{B\left(x_{0}, r\right)}\left|\eta-\eta\left(x_{0}\right)\right| d \mu^{s}<R \varepsilon
\end{gathered}
$$

by (3.13). Since $\left\langle f, \nu_{x}\right\rangle \in L^{1}(\Omega, \mu)$ by (2.5) , excluding a further $\mu^{s}$-negligible set and possibly making $r>0$ smaller, we can also assume that

$$
f_{B\left(x_{0}, r\right)}\left|\left\langle f, \nu_{x}\right\rangle-\left\langle f, \nu_{x_{0}}\right\rangle\right| d \mu<\varepsilon
$$

Clearly $f^{\infty} \equiv 0$ and then by (3.11), we have

$$
f\left(\frac{d D u_{j}}{d \mu}\right) \mu \stackrel{*}{\rightarrow}\left\langle f, \nu_{x}\right\rangle a \mathcal{L}^{n}+\left\langle f, \nu_{x}\right\rangle \mu^{s} \quad \text { in } \mathcal{M}(\Omega) .
$$


Now by (3.15),

$$
\begin{aligned}
\left\langle f, \nu_{x_{0}}\right\rangle & \leq f_{B\left(x_{0}, r\right)}\left\langle f, \nu_{x}\right\rangle d \mu+\varepsilon \\
& \leq \liminf _{j \rightarrow \infty} f_{B\left(x_{0}, r\right)} f\left(\frac{d D u_{j}}{d \mu}\right) d \mu+\varepsilon \\
& \stackrel{f}{\leq 1} \liminf _{j \rightarrow \infty} f_{B\left(x_{0}, r\right)} f\left(\frac{d D u_{j}}{d \mu^{s}}\right) d \mu^{s}+\frac{\int_{B\left(x_{0}, r\right)} a d \mathcal{L}^{n}}{\mu\left(B\left(x_{0}, r\right)\right)}+\varepsilon \\
& \stackrel{(3.13)}{\leq} \liminf _{j \rightarrow \infty} f_{B\left(x_{0}, r\right)} f\left(\frac{d D u_{j}}{d \mu^{s}}\right) d \mu^{s}+2 \varepsilon \\
& \stackrel{(3.12)}{=} \liminf _{j \rightarrow \infty} f_{B\left(x_{0}, r\right)} f\left(\xi_{j} \otimes \eta\right) d \mu^{s}+2 \varepsilon \\
& \stackrel{(3.14)}{\leq} \liminf _{j \rightarrow \infty} f_{B\left(x_{0}, r\right)} f\left(\xi_{j} \otimes \eta\left(x_{0}\right)\right) d \mu^{s}+R \varepsilon+2 \varepsilon \\
& \leq 3 R \varepsilon,
\end{aligned}
$$

since $f$ is zero on the hyperplane $\mathbb{R}^{N} \otimes \eta\left(x_{0}\right)$. Letting $\varepsilon \rightarrow 0$, we get $\left\langle f, \nu_{x_{0}}\right\rangle=$ 0 , implying that $\nu_{x_{0}}$ is carried on the set $\left(\mathbb{R}^{N} \otimes \eta\left(x_{0}\right)\right) \cup B(0, R)^{c}$. Letting $R \rightarrow \infty$, we obtain that $\nu_{x_{0}}$ is carried on the hyperplane $\mathbb{R}^{N} \otimes \eta\left(x_{0}\right)$.

By choosing $f$ to be the identity mapping on $\mathbb{R}^{N \times n}$ in (3.11) (componentwise, to be precise), and noting that $D u_{j} \stackrel{*}{\rightarrow} D u$ in $\mathcal{M}(\Omega)$ (the fact that $\left(u_{j}\right)$ is a norm-bounded sequence in $\operatorname{BV}\left(\Omega ; \mathbb{R}^{N}\right)$ implies that $u_{j} \stackrel{*}{\rightarrow} u$ in $\left.\operatorname{BV}\left(\Omega ; \mathbb{R}^{N}\right)\right)$, we get for the singular parts

$$
D^{s} u=\left\langle\mathrm{id}, \nu_{x}\right\rangle \mu^{s}+\left\langle\mathrm{id}, \nu_{x}^{\infty}\right\rangle \lambda_{\nu}^{s}
$$

in $\Omega$. Using the fact that $\left\langle\mathrm{id}, \nu_{x}\right\rangle \in \mathbb{R}^{N} \otimes \eta(x)$ for $\mu^{s}$-almost every $x \in \Omega$, we get

$$
\left\langle\mathrm{id}, \nu_{x}^{\infty}\right\rangle \frac{d \lambda_{\nu}^{s}}{d \mu^{s}}=\frac{d D^{s} u}{d \mu^{s}}(x)-\left\langle\mathrm{id}, \nu_{x}\right\rangle=\xi(x) \otimes \eta(x)-\left\langle\mathrm{id}, \nu_{x}\right\rangle \in \mathbb{R}^{N} \otimes \eta(x)
$$

for $\mu^{s}$-almost every $x \in \Omega$. Since $F^{\infty}$ is quasiconvex and 1-homogenous, we have $F^{\infty}(A)=\left(F^{\infty}\right)^{c}(A)$ for all rank one $A \in \mathbb{R}^{N \times n}$, where the convex envelope is defined by

$$
G^{c}(A):=\sup \{H(A): H \text { convex, } H \leq G\},
$$

see [12, Corollary 1.2]. According to [2, Lemma 5.5 (i)], for any convex function $g: \mathbb{R}^{N \times n} \rightarrow \mathbb{R}$ we have

$$
g\left(A_{1}+A_{2}\right) \leq g\left(A_{1}\right)+g^{\infty}\left(A_{2}\right)
$$


for all $A_{1}, A_{2} \in \mathbb{R}^{N \times n}$. Note that in (3.16), all three terms belong to $\mathbb{R}^{N} \otimes \eta(x)$ for $\mu^{s}$-almost every $x \in \Omega$. Since $\xi \mapsto F(\xi \otimes \eta(x))$ is convex for a fixed $x \in \Omega$ by the rank one convexity of $F$, we get by (3.17)

$$
\begin{aligned}
& F\left(\frac{d D^{s} u}{d \mu^{s}}(x)\right) \leq F\left(\left\langle\mathrm{id}, \nu_{x}\right\rangle\right)+F^{\infty}\left(\left\langle\mathrm{id}, \nu_{x}^{\infty}\right\rangle\right) \frac{d \lambda_{\nu}^{s}}{d \mu^{s}} \\
& =F\left(\left\langle\mathrm{id}, \nu_{x}\right\rangle\right)+\left(F^{\infty}\right)^{c}\left(\left\langle\mathrm{id}, \nu_{x}^{\infty}\right\rangle\right) \frac{d \lambda_{\nu}^{s}}{d \mu^{s}} \\
& \stackrel{\text { Jensen }}{\leq}\left\langle F, \nu_{x}\right\rangle+\left\langle\left(F^{\infty}\right)^{c}, \nu_{x}^{\infty}\right\rangle \frac{d \lambda_{\nu}^{s}}{d \mu^{s}} \\
& \leq\left\langle F, \nu_{x}\right\rangle+\left\langle F^{\infty}, \nu_{x}^{\infty}\right\rangle \frac{d \lambda_{\nu}^{s}}{d \mu^{s}}
\end{aligned}
$$

for $\mu^{s}$-almost every $x \in \Omega$. Combining this with (3.11) - note that $F \in$ $\mathbf{S Q}\left(\mathbb{R}^{N \times n}\right) \subset \mathbf{E}\left(\Omega ; \mathbb{R}^{N \times n}\right)$ - we get for any ball $B(y, r) \subset \Omega$

$$
\int_{B(y, r)} F\left(\frac{d D^{s} u}{d \mu^{s}}\right) d \mu^{s} \leq \liminf _{j \rightarrow \infty} \int_{B(y, r)} F\left(\frac{d D u_{j}}{d \mu}\right) d \mu .
$$

\subsubsection{Combining the estimates}

Now we combine the previous two lemmas to prove Proposition 3.2 .

Proof of Proposition 3.2. We keep assuming that $F \in \mathbf{S Q}\left(\mathbb{R}^{N \times n}\right)$ with parameters $i, r_{i} \geq 1$ and linear growth $0 \leq F(A) \leq M(1+|A|)$. Let $\left(u_{j}\right) \subset$ $W_{\mu}^{1,1}\left(\Omega ; \mathbb{R}^{N}\right)$ be a sequence with $u_{j} \rightarrow u$ in $L^{1}\left(\Omega ; \mathbb{R}^{N}\right)$, and we can also assume that (3.3) holds, so that the assumptions of both Lemma 3.3 and Lemma 3.4 are satisfied.

Fix $\varepsilon>0$. Let $H \subset \Omega$ be a Borel set with $\mathcal{L}^{n}(H)=0$ and $\mu^{s}(\Omega \backslash H)=0$. Also, let $D \subset \Omega$ be a Borel set with $\mu(D)=0$ and $\left|D^{s, \mu} u\right|(\Omega \backslash D)=0$. Take an open set $G \subset \Omega$ with $G \supset H \backslash D$ and

$$
\left|D^{s, \mu} u\right|(G)+\int_{G} M(a+|\nabla u|) d \mathcal{L}^{n}<\varepsilon
$$

Consider the fine cover $\{\bar{B}(x, R)\}_{x \in H \backslash D}$ of the set $H \backslash D$, with the balls $\bar{B}(x, R)$ contained in $G$ and satisfying $\mu^{s}(\partial B(x, R))=0$. By Vitali's covering theorem, we can pick a countable, disjoint collection $\left\{B_{i}\right\}_{i \in \mathbb{N}}:=\left\{B\left(x_{i}, R_{i}\right)\right\}_{i \in \mathbb{N}}$ with

$$
\mu^{s}\left((H \backslash D) \backslash \bigcup_{i=1}^{\infty} B_{i}\right)=0 \text { and thus } \mu^{s}\left(\Omega \backslash \bigcup_{i=1}^{\infty} B_{i}\right)=0
$$


Pick also $m \in \mathbb{N}$ such that

$$
\mu\left(\bigcup_{i=m}^{\infty} \bar{B}_{i}\right)+M \int_{\bigcup_{i=m}^{\infty} \bar{B}_{i}}\left(1+\frac{d\left|D^{s} u\right|}{d \mu^{s}}\right) d \mu^{s}<\varepsilon .
$$

By (3.10) we have

$$
\begin{aligned}
\liminf _{j \rightarrow \infty} \int_{\bigcup_{i=1}^{m} B_{i}} F\left(\frac{d D u_{j}}{d \mu}\right) d \mu & \geq \int_{\bigcup_{i=1}^{m} B_{i}} F\left(\frac{d D^{s} u}{d \mu^{s}}\right) d \mu^{s} \\
& \geq \int_{\Omega} F\left(\frac{d D^{s} u}{d \mu^{s}}\right) d \mu^{s}-\varepsilon
\end{aligned}
$$

by (3.20) and the linear growth of $F$.

By combining (3.19) and (3.20), we get

$$
\mu^{s}\left(\Omega \backslash \bigcup_{i=1}^{m} \bar{B}_{i}\right)<\varepsilon .
$$

Moreover, we can write (3.4) with the choice $U=\Omega \backslash \bigcup_{i=1}^{m} \overline{B_{i}}$ :

$$
\begin{aligned}
& \liminf _{j \rightarrow \infty} \int_{\Omega \backslash \bigcup_{i=1}^{m} \bar{B}_{i}} F\left(\frac{d D u_{j}}{d \mu}\right) d \mu \\
& \geq \int_{\Omega \backslash \bigcup_{i=1}^{m} \bar{B}_{i}} F\left(\frac{\nabla u}{a}\right) a d \mathcal{L}^{n}+\int_{\Omega \backslash \bigcup_{i=1}^{m} \bar{B}_{i}} F^{\infty}\left(\frac{d D^{s} u}{d\left|D^{s} u\right|}\right) d\left|D^{s} u\right| \\
& \quad-\left(M r_{i}+i\right) \mu^{s}\left(\Omega \backslash \bigcup_{i=1}^{m} \bar{B}_{i}\right) \\
& \quad \int_{\Omega \backslash \bigcup_{i=1}^{m} \bar{B}_{i}} F\left(\frac{\nabla u}{a}\right) a d \mathcal{L}^{n}+\int_{\Omega \backslash \bigcup_{i=1}^{m} \bar{B}_{i}} F^{\infty}\left(\frac{d D^{s, \mu} u}{d\left|D^{s, \mu} u\right|}\right) d\left|D^{s, \mu} u\right| \\
& \quad-\left(M r_{i}+i\right) \varepsilon \\
& \quad \int_{\Omega \backslash \bigcup_{i=1}^{m} \bar{B}_{i}} F\left(\frac{\nabla u}{a}\right) a d \mathcal{L}^{n}+\int_{\Omega} F^{\infty}\left(\frac{d D^{s, \mu} u}{d\left|D^{s, \mu} u\right|}\right) d\left|D^{s, \mu} u\right| \\
& \quad-M \varepsilon-\left(M r_{i}+i\right) \varepsilon \\
& \frac{(3.18 \mid}{\geq} \int_{\Omega} F\left(\frac{\nabla u}{a}\right) a d \mathcal{L}^{n}+\int_{\Omega} F^{\infty}\left(\frac{d D^{s, \mu} u}{d\left|D^{s, \mu} u\right|}\right) d\left|D^{s, \mu} u\right| \\
& \quad-\varepsilon-M \varepsilon-\left(M r_{i}+i\right) \varepsilon .
\end{aligned}
$$


Combining this with (3.21), we get

$$
\begin{aligned}
\liminf _{j \rightarrow \infty} \int_{\Omega} F & \left(\frac{d D u_{j}}{d \mu}\right) d \mu \\
\geq & \int_{\Omega} F\left(\frac{\nabla u}{a}\right) a d \mathcal{L}^{n}+\int_{\Omega} F^{\infty}\left(\frac{d D^{s, \mu} u}{d\left|D^{s, \mu} u\right|}\right) d\left|D^{s, \mu} u\right| \\
& \quad+\int_{\bigcup_{i=1}^{m} B_{i}} F\left(\frac{d D^{s} u}{d \mu^{s}}\right) d \mu^{s}-3\left(M r_{i}+i\right) \varepsilon \\
\frac{3.20)}{\geq} & \int_{\Omega} F\left(\frac{\nabla u}{a}\right) a d \mathcal{L}^{n}+\int_{\Omega} F^{\infty}\left(\frac{d D^{s, \mu} u}{d\left|D^{s, \mu} u\right|}\right) d\left|D^{s, \mu} u\right| \\
& +\int_{\Omega} F\left(\frac{d D^{s} u}{d \mu^{s}}\right) d \mu^{s}-4\left(M r_{i}+i\right) \varepsilon .
\end{aligned}
$$

By letting $\varepsilon \rightarrow 0$, we get the estimate from below.

Finally, we remove the assumption $F \in \mathbf{S Q}\left(\mathbb{R}^{N \times n}\right)$. By [12, Lemma 6.3], we can find a sequence $F_{i} \in \mathbf{S Q}\left(\mathbb{R}^{N \times n}\right)$ with $F_{i}(A) \searrow F(A)$ and $F_{i}^{\infty}(A) \searrow$ $F^{\infty}(A)$ pointwise for every $A \in \mathbb{R}^{N \times n}$ as $i \rightarrow \infty$, and by making $M$ slightly larger, if necessary, we can also assume that $m|A| \leq F_{i}(A) \leq M(1+|A|)$ for every $i \in \mathbb{N}$.

As before, let $\left(u_{j}\right) \subset W_{\mu}^{1,1}\left(\Omega ; \mathbb{R}^{N}\right)$ with $u_{j} \rightarrow u$ in $L^{1}\left(\Omega ; \mathbb{R}^{N}\right)$. We can again assume that (3.3) holds, and by the coercivity $m|A| \leq F(A)$, this implies that $\left(u_{j}\right)$ is a norm-bounded sequence in $\operatorname{BV}\left(\Omega ; \mathbb{R}^{N}\right)$. Thus by Theorem 2.3. a subsequence of $D u_{j}$ (not relabeled) generates a generalized Young measure $\left(\nu_{x}, \lambda_{\nu}, \nu_{x}^{\infty}\right)$ with respect to $\mu$. Thus we have for any $i \in \mathbb{N}$

$$
\begin{aligned}
\int_{\Omega} F\left(\frac{d D u}{d \mu}\right) d \mu & \int_{\Omega} F^{\infty}\left(\frac{d D^{s, \mu} u}{d\left|D^{s, \mu} u\right|}\right) d\left|D^{s, \mu} u\right| \\
& \leq \int_{\Omega} F_{i}\left(\frac{d D u}{d \mu}\right) d \mu+\int_{\Omega} F_{i}^{\infty}\left(\frac{d D^{s, \mu} u}{d\left|D^{s, \mu} u\right|}\right) d\left|D^{s, \mu} u\right| \\
& \leq \liminf _{j \rightarrow \infty} \int_{\Omega} F_{i}\left(\frac{d D u_{j}}{d \mu}\right) d \mu \\
& =\int_{\Omega}\left\langle F_{i}, \nu_{x}\right\rangle d \mu+\int_{\bar{\Omega}}\left\langle F_{i}^{\infty}, \nu_{x}^{\infty}\right\rangle d \lambda_{\nu} .
\end{aligned}
$$

On the other hand, by Lebesgue's dominated convergence theorem, as well as the fact that $F \in \mathbf{Q}\left(\mathbb{R}^{N \times n}\right) \cap \mathbf{R}\left(\Omega ; \mathbb{R}^{N \times n}\right) \subset \mathbf{E}\left(\Omega ; \mathbb{R}^{N \times n}\right)$,

$$
\begin{aligned}
\lim _{i \rightarrow \infty}\left(\int_{\Omega}\left\langle F_{i}, \nu_{x}\right\rangle d \mu+\int_{\bar{\Omega}}\left\langle F_{i}^{\infty}, \nu_{x}^{\infty}\right\rangle d \lambda_{\nu}\right) & =\int_{\Omega}\left\langle F, \nu_{x}\right\rangle d \mu+\int_{\bar{\Omega}}\left\langle F^{\infty}, \nu_{x}^{\infty}\right\rangle d \lambda_{\nu} \\
& =\lim _{j \rightarrow \infty} \int_{\Omega} F\left(\frac{d D u_{j}}{d \mu}\right) d \mu .
\end{aligned}
$$


By combining (3.23) and (3.24), we get the desired estimate from below.

\subsection{Estimate from above}

Recall from (3.1) the definition of the functional $\mathcal{F}_{*}$ by relaxation. We prove that the estimate from above holds for the integral representation of $\mathcal{F}_{*}$. Here our proof is not based on the theory of Young measures, so we can allow for somewhat weaker assumptions on $F$.

Proposition 3.5. Let $\Omega \subset \mathbb{R}^{n}$ be a bounded open set, let $\mu \in \mathcal{M}^{+}(\Omega)$ with $\mathcal{L}^{n} \ll \mu$, let $F \in \mathbf{Q}\left(\mathbb{R}^{N \times n}\right)$ with

$$
0 \leq F(A) \leq M(1+|A|), \quad A \in \mathbb{R}^{N \times n}
$$

for some $M \geq 1$, and let $u \in \operatorname{BV}\left(\Omega ; \mathbb{R}^{N}\right)$. Then we have

$$
\mathcal{F}_{*}(u, \Omega) \leq \int_{\Omega} F\left(\frac{d D u}{d \mu}\right) d \mu+\int_{\Omega} F^{\infty}\left(\frac{d D^{s, \mu} u}{d\left|D^{s, \mu} u\right|}\right) d\left|D^{s, \mu} u\right| .
$$

Proof. Again, by Definition 3.1 and [12, Lemma 6.3] we can find a sequence $F_{i} \in \mathbf{S Q}\left(\mathbb{R}^{N \times n}\right)$ with parameters $i \in \mathbb{N}, r_{i}>0$ such that $F_{i}(A) \searrow F(A)$ and $F_{i}^{\infty}(A) \searrow F^{\infty}(A)$ pointwise for every $A \in \mathbb{R}^{N \times n}$ as $i \rightarrow \infty$. Moreover, by making $M$ slightly larger, if necessary, we have that $0 \leq F_{i}(A) \leq M(1+|A|)$ for all $i \in \mathbb{N}$ and $A \in \mathbb{R}^{N \times n}$. Fix $i \in \mathbb{N}$.

The proof is based on mollifying the function $u$ in a small set. Take a Borel set $D \subset \Omega$ with $\left|D^{s, \mu} u\right|(\Omega \backslash D)=0$ and $\mu(D)=0$. Then take an open set $G \supset D$ with $\mathcal{L}^{n}(G)$ and $\mu(G)$ so small that

$$
\int_{G} M(1+|\nabla u|) d \mathcal{L}^{n}+\int_{G} M\left|\frac{d D^{s} u}{d \mu}\right| d \mu+M\left(r_{i}+i\right) \mathcal{L}^{n}(G)+M\left(1+r_{i}\right) \mu(G)
$$

is less than $1 / i$; this is possible by the absolute continuity of integrals. By Lemma 2.1 we can pick a sequence $\left(v_{j}\right) \subset \mathrm{BV}_{u}\left(G ; \mathbb{R}^{N}\right) \cap C^{\infty}\left(G ; \mathbb{R}^{N}\right.$ ) (note boundary values) that converges to $u\langle\cdot\rangle$-strictly in $\operatorname{BV}\left(G ; \mathbb{R}^{N}\right)$. Fix also $j \in \mathbb{N}$.

Using the linear growth of $F_{i}$, we estimate

$$
\begin{aligned}
\int_{G} F\left(\frac{d D v_{j}}{d \mu}\right) d \mu & \leq \int_{G} F_{i}\left(\frac{d D v_{j}}{d \mu}\right) d \mu=\int_{G} F_{i}\left(\frac{\nabla v_{j}}{a}\right) a d \mathcal{L}^{n} \\
& \leq \int_{G \cap\left\{\left|\nabla v_{j} / a\right|>r_{i}\right\}} F_{i}\left(\frac{\nabla v_{j}}{a}\right) a d \mathcal{L}^{n}+M\left(1+r_{i}\right) \mu(G),
\end{aligned}
$$


where by the fact that $F(A)=F^{\infty}(A)-i$ for $|A| \geq r_{i}$, the last integral equals

$$
\begin{aligned}
\int_{G \cap\left\{\left|\nabla v_{j} / a\right|>r_{i}\right\}}\left(F_{i}^{\infty}\left(\frac{\nabla v_{j}}{a}\right)-i\right) a d \mathcal{L}^{n} \leq \int_{G \cap\left\{\left|\nabla v_{j} / a\right|>r_{i}\right\}} F_{i}^{\infty}\left(\frac{\nabla v_{j}}{a}\right) a d \mathcal{L}^{n} \\
=\int_{G \cap\left\{\left|\nabla v_{j} / a\right|>r_{i}\right\}} F_{i}^{\infty}\left(\nabla v_{j}\right) d \mathcal{L}^{n} \\
\leq \int_{G} F_{i}^{\infty}\left(\nabla v_{j}\right) d \mathcal{L}^{n} \\
\leq \int_{G \cap\left\{\left|\nabla v_{j}\right|>r_{i}\right\}} F_{i}^{\infty}\left(\nabla v_{j}\right) d \mathcal{L}^{n}+M r_{i} \mathcal{L}^{n}(G) \\
=\int_{G \cap\left\{\left|\nabla v_{j}\right|>r_{i}\right\}}\left(F_{i}\left(\nabla v_{j}\right)+i\right) d \mathcal{L}^{n}+M r_{i} \mathcal{L}^{n}(G) \\
\leq \int_{G} F_{i}\left(\nabla v_{j}\right) d \mathcal{L}^{n}+M\left(r_{i}+i\right) \mathcal{L}^{n}(G) .
\end{aligned}
$$

Now, since $F_{i} \in \mathbf{S Q}\left(\mathbb{R}^{N \times n}\right) \subset \mathbf{E}\left(G ; \mathbb{R}^{N \times n}\right)$ (constant in the $x$-variable) and $v_{j} \rightarrow u\langle\cdot\rangle$-strictly in $\operatorname{BV}\left(G ; \mathbb{R}^{N}\right)$, we can apply Reshetnyak's continuity theorem, Theorem [2.2, to obtain

$$
\begin{aligned}
\liminf _{j \rightarrow \infty} & \int_{G} F\left(\frac{d D v_{j}}{d \mu}\right) d \mu \\
\leq & \liminf _{j \rightarrow \infty} \int_{G} F_{i}\left(\nabla v_{j}\right) d \mathcal{L}^{n}+M\left(r_{i}+i\right) \mathcal{L}^{n}(G)+M\left(1+r_{i}\right) \mu(G) \\
= & \int_{G} F_{i}(\nabla u) d \mathcal{L}^{n}+\int_{G} F_{i}^{\infty}\left(\frac{d D^{s} u}{d\left|D^{s} u\right|}\right) d\left|D^{s} u\right| \\
& +M\left(r_{i}+i\right) \mathcal{L}^{n}(G)+M\left(1+r_{i}\right) \mu(G) \\
\leq & \int_{G} M(1+|\nabla u|) d \mathcal{L}^{n}+\int_{G} M\left|\frac{d D^{s} u}{d \mu}\right| d \mu \\
& +\int_{G} F_{i}^{\infty}\left(\frac{d D^{s, \mu} u}{d\left|D^{s \mu} u\right|}\right) d\left|D^{s, \mu} u\right|+M\left(r_{i}+i\right) \mathcal{L}^{n}(G)+M\left(1+r_{i}\right) \mu(G) \\
\leq & \int_{G} F_{i}^{\infty}\left(\frac{d D^{s, \mu} u}{d\left|D^{s \mu} u\right|}\right) d\left|D^{s, \mu} u\right|+1 / i
\end{aligned}
$$

by (3.25). Then define for each $j \in \mathbb{N}$

$$
u_{j}:= \begin{cases}v_{j} & \text { in } G, \\ u & \text { in } \Omega \backslash G .\end{cases}
$$

The fact that $v_{j} \in \mathrm{BV}_{u}\left(G ; \mathbb{R}^{N}\right)$ implies by definition (given before Lemma 2.1) that $D u_{j}=D u\left\llcorner\Omega \backslash G+D v_{j}\left\llcorner G\right.\right.$. Thus it is clear that $u_{j} \in W_{\mu}^{1,1}\left(\Omega ; \mathbb{R}^{N}\right)$, 
and also $u_{j} \rightarrow u$ in $L^{1}\left(\Omega ; \mathbb{R}^{N}\right)$, so that $u_{j}$ is an admissible sequence for $\mathcal{F}_{*}(u, \Omega)$. In total, we obtain

$$
\begin{aligned}
\mathcal{F}_{*}(u, \Omega) & \leq \liminf _{j \rightarrow \infty} \int_{\Omega} F\left(\frac{d D u_{j}}{d \mu}\right) d \mu \\
& =\liminf _{j \rightarrow \infty} \int_{G} F\left(\frac{d D v_{j}}{d \mu}\right) d \mu+\int_{\Omega \backslash G} F\left(\frac{d D u}{d \mu}\right) d \mu \\
& \leq \int_{G} F_{i}^{\infty}\left(\frac{d D^{s, \mu} u}{d\left|D^{s \mu} u\right|}\right) d\left|D^{s, \mu} u\right|+\int_{\Omega \backslash G} F\left(\frac{d D u}{d \mu}\right) d \mu+1 / i \\
& \leq \int_{\Omega} F_{i}\left(\frac{d D u}{d \mu}\right) d \mu+\int_{\Omega} F_{i}^{\infty}\left(\frac{d D^{s, \mu} u}{d\left|D^{s \mu} u\right|}\right) d\left|D^{s, \mu} u\right|+1 / i
\end{aligned}
$$

Letting $i \rightarrow \infty$, by Lebesgue's monotone or dominated convergence we get the desired estimate from above.

\subsection{Some examples}

Let us briefly consider why it is necessary to assume that $\mathcal{L}^{n} \ll \mu$, at least in order to obtain the integral representation (3.2). The reason is that the estimate from above may be violated without this assumption. We note that the integral representation (3.2) always takes a value at most

$$
M \mu(\Omega)+M|D u|(\Omega),
$$

which is finite for a $\mathrm{BV}$ function $u \in \operatorname{BV}\left(\Omega ; \mathbb{R}^{N}\right)$. On the other hand, if it is not true that $\mathcal{L}^{n} \ll \mu$, then there can be a large set not "seen" by the measure $\mu$, and as a result it may simply be impossible to approximate certain BV functions in the $L^{1}$-sense by functions in the class $W_{\mu}^{1,1}\left(\Omega ; \mathbb{R}^{N}\right)$. Consider the following examples.

Example 3.6. Suppose that there is an open set $B \subset \Omega$ (which we can assume to be a ball) with $\mu(B)=0$ but of course $\mathcal{L}^{n}(B)>0$. Take a nonconstant $u \in C_{c}^{1}(B)$, and note that all functions $u_{j} \in W_{\mu}^{1,1}(\Omega)$ satisfy $\left|D u_{j}\right|(B)=0$ and are thus constant in the ball $B$. Thus there is no sequence of functions $u_{j} \in W_{\mu}^{1,1}(\Omega)$ with $u_{j} \rightarrow u$ in $L^{1}(\Omega)$, and consequently $\mathcal{F}_{*}(u, \Omega)=\infty$.

Even if the support of $\mu$ is the whole of $\bar{\Omega}$, the estimate from above may fail.

Example 3.7. Take $\Omega$ to be the open unit square on the plane, and let $A \subset \Omega$ be a "fat" Sierpinski carpet, with $\mathcal{L}^{2}(A)=1 / 2$. Then define the 
weight $w=\mathbb{1}_{\Omega \backslash A}$, and $\mu:=w \mathcal{L}^{2}$. Clearly the absolute continuity assumption $\mathcal{L}^{2} \ll \mu$ is violated, but the support of $\mu$ is the whole of $\bar{\Omega}$. By using the properties of BV functions restricted to lines, see e.g. [5, Section 3.11], we obtain that any function $v \in W_{\mu}^{1,1}(\Omega)$ is constant almost everywhere in $A$. If we define a BV function $u \in \mathrm{BV}(\Omega)$ e.g. as $u(x, y):=x$, there is no sequence $u_{j} \in W_{\mu}^{1,1}(\Omega)$ for which $u_{j} \rightarrow u$ in $L^{1}(\Omega)$, and consequently $\mathcal{F}_{*}(u, \Omega)=\infty$.

However, it is not clear to us whether the assumption $\mathcal{L}^{n} \ll \mu$, or the assumption on the integrand $F \in \mathbf{R}\left(\Omega ; \mathbb{R}^{N \times n}\right)$, are necessary in our main result, Theorem 1.1 .

\section{The lower semicontinuity theorem}

From the integral representation, we obtain the following lower semicontinuity result.

Proposition 4.1. Let $\Omega \subset \mathbb{R}^{n}$ be a bounded open set with $\mathcal{L}^{n}(\partial \Omega)=0$, let $\mu \in \mathcal{M}^{+}(\Omega)$ with $\mathcal{L}^{n} \ll \mu$, and let $F \in \mathbf{R}\left(\Omega ; \mathbb{R}^{N \times n}\right) \cap \mathbf{Q}\left(\mathbb{R}^{N \times n}\right)$ with

$$
m|A| \leq F(A) \leq M(1+|A|)
$$

for some $0<m \leq M$. Then the functional

$\mathcal{F}(u):=\int_{\Omega} F\left(\frac{d D u}{d \mu}\right) d \mu+\int_{\Omega} F^{\infty}\left(\frac{d D^{s, \mu} u}{d\left|D^{s, \mu} u\right|}\right) d\left|D^{s, \mu} u\right|, \quad u \in \operatorname{BV}\left(\Omega ; \mathbb{R}^{N}\right)$,

is lower semicontinuous with respect to convergence in $L^{1}\left(\Omega ; \mathbb{R}^{N}\right)$.

Proof. The relaxed functional $\mathcal{F}_{*}(u, \Omega)$ given in (3.1) is obviously lower semicontinuous with respect to convergence in $L^{1}\left(\Omega ; \mathbb{R}^{N}\right)$, and by Proposition 3.2 and Proposition 3.5 it equals the functional $\mathcal{F}(u)$ given in this proposition.

We recall Jensen's inequalities for gradient Young measures with respect to the Lebesgue measure $\mathcal{L}^{n}$, given in Theorem 2.5. We can now partially generalize these inequalities to the case of a general measure $\mu$.

Theorem 4.2. Let $\Omega \subset \mathbb{R}^{n}$ be a bounded open set with $\mathcal{L}^{n}(\partial \Omega)=0$, let $\mu \in \mathcal{M}^{+}(\Omega)$ with $\mathcal{L}^{n} \ll \mu$, let $F \in \mathbf{R}\left(\Omega ; \mathbb{R}^{N \times n}\right) \cap \mathbf{Q}\left(\mathbb{R}^{N \times n}\right)$ with $F \geq 0$, let $u \in \operatorname{BV}\left(\Omega ; \mathbb{R}^{N}\right)$, and let $\nu \in \mathbf{Y}\left(\Omega, \mu ; \mathbb{R}^{N \times n}\right)$ be a gradient Young measure with $\lambda_{\nu}(\partial \Omega)=0$ and with barycenter Du. Then the following hold:

$$
\begin{aligned}
& F\left(\frac{d D u}{d \mu}\right) \leq\left\langle F, \nu_{x}\right\rangle+\left\langle F^{\infty}, \nu_{x}^{\infty}\right\rangle \frac{d \lambda_{\nu}}{d \mu}(x) \quad \text { for } \mu \text {-almost every } x \in \Omega, \\
& F^{\infty}\left(\frac{d D^{s, \mu} u}{d\left|D^{s, \mu} u\right|}\right)\left|D^{s, \mu} u\right| \leq\left\langle F^{\infty}, \nu_{x}^{\infty}\right\rangle \lambda_{\nu}^{s, \mu} \quad \text { as measures. }
\end{aligned}
$$


Proof. Take a sequence $\left(u_{j}\right) \subset \mathrm{BV}\left(\Omega ; \mathbb{R}^{N}\right)$ that generates $\nu$. We know that $u_{j} \stackrel{*}{\rightarrow} u$ in $\operatorname{BV}\left(\Omega ; \mathbb{R}^{N}\right)$, see the discussion after (2.7). Note that $F \in$ $\mathbf{R}\left(\Omega ; \mathbb{R}^{N \times n}\right) \cap \mathbf{Q}\left(\mathbb{R}^{N \times n}\right) \subset \mathbf{E}\left(\Omega ; \mathbb{R}^{N \times n}\right)$ (constant in the $x$-variable), so that $F$ necessarily has linear growth $F(A) \leq M(1+|A|)$ for some $M \geq 0$. Let us first also assume that $F$ has the coercivity property $m|A| \leq F(A)$ for some $m>0$ and all $A \in \mathbb{R}^{N \times n}$. By combining our lower semicontinuity result, Proposition 4.1, with the fact that $F \in \mathbf{E}\left(\Omega ; \mathbb{R}^{N \times n}\right)$, we obtain

$$
\begin{gathered}
\int_{\Omega} F\left(\frac{d D u}{d \mu}\right) d \mu+\int_{\Omega} F^{\infty}\left(\frac{d D^{s, \mu} u}{d\left|D^{s, \mu} u\right|}\right) d\left|D^{s, \mu} u\right| \\
\leq \liminf _{j \rightarrow \infty}\left(\int_{\Omega} F\left(\frac{d D u_{j}}{d \mu}\right) d \mu+\int_{\Omega} F^{\infty}\left(\frac{d D^{s, \mu} u_{j}}{d\left|D^{s, \mu} u_{j}\right|}\right) d\left|D^{s, \mu} u_{j}\right|\right) \\
=\int_{\Omega}\left\langle F_{i}, \nu_{x}\right\rangle d \mu+\int_{\Omega}\left\langle F_{i}^{\infty}, \nu_{x}^{\infty}\right\rangle d \lambda_{\nu} .
\end{gathered}
$$

We can equally well write the above inequality in any open $U \subset \Omega$ (in particular, a ball) with $\lambda_{\nu}(\partial U)=0$. Thus we can differentiate the inequality with respect to $\mu$, and obtain (4.1) by the Besicovitch differentiation theorem (see e.g. [5, Theorem 2.22]). By writing the above inequality for balls from a suitable Vitali covering of $\Omega$, we obtain (4.2).

The general case can be obtained by writing (4.1) and (4.2) for integrands

$$
F_{i}(A):=\max \{F(A),|A| / i\}, \quad i \in \mathbb{N},
$$

and letting $i \rightarrow \infty$.

Corollary 4.3. With $\Omega, \mu, u$, and $\nu$ as in the previous theorem, there exist sets $E_{1}, E_{2} \subset \Omega$ with $\mu\left(E_{1}\right)=0$ and $\left|D^{s, \mu} u\right|\left(E_{2}\right)=0$ such that for every $F \in \mathbf{R}\left(\Omega ; \mathbb{R}^{N \times n}\right) \cap \mathbf{Q}\left(\mathbb{R}^{N \times n}\right)$ with $F \geq 0$, we have

$$
\begin{gathered}
F\left(\frac{d D u}{d \mu}\right) \leq\left\langle F, \nu_{x}\right\rangle+\left\langle F^{\infty}, \nu_{x}^{\infty}\right\rangle \frac{d \lambda_{\nu}}{d \mu}(x) \quad \text { for every } x \in \Omega \backslash E_{1}, \\
F^{\infty}\left(\frac{d D^{s, \mu} u}{d\left|D^{s, \mu} u\right|}\right) \leq\left\langle F^{\infty}, \nu_{x}^{\infty}\right\rangle \frac{d \lambda_{\nu}^{s, \mu}}{d\left|D^{s, \mu} u\right|} \quad \text { for every } x \in \Omega \backslash E_{2} .
\end{gathered}
$$

The point is that we can find exceptional sets that do not depend on the integrand $F$.

Proof. Again, we note that $\mathbf{R}\left(\Omega ; \mathbb{R}^{N \times n}\right) \cap \mathbf{Q}\left(\mathbb{R}^{N \times n}\right) \subset \mathbf{E}\left(\Omega ; \mathbb{R}^{N \times n}\right)$ (constant in the $x$-variable). Recalling the transformation $T$ given in Section 2.3 , we have that

$$
\left\{T(F): F \in \mathbf{R}\left(\Omega ; \mathbb{R}^{N \times n}\right) \cap \mathbf{Q}\left(\mathbb{R}^{N \times n}\right), F \geq 0\right\}
$$


contains a countable dense subset $\left\{G_{i}\right\}_{i \in \mathbb{N}}$, since it is contained in the separable space $C\left(\overline{\mathbb{B}^{N \times m}}\right)$. Then (4.3) and (4.4) hold for some choice of sets $E_{1}, E_{2} \subset \Omega$ with $\mu\left(E_{1}\right)=0$ and $\left|D^{s, \mu} u\right|\left(E_{2}\right)=0$, and with $F=T^{-1} G_{i}$ for any $i \in \mathbb{N}$. It is easy to see for any $F \in \mathbf{R}\left(\Omega ; \mathbb{R}^{N \times n}\right) \cap \mathbf{Q}\left(\mathbb{R}^{N \times n}\right), F \geq 0$ that

$$
F_{k}\left(\frac{d D u}{d \mu}(x)\right)-F\left(\frac{d D u}{d \mu}(x)\right) \rightarrow 0
$$

for every $x \in \Omega \backslash E_{1}$, for a sequence $\left(F_{k}\right) \subset\left\{T^{-1} G_{i}\right\}_{i \in \mathbb{N}}$ with $T\left(F_{k}\right) \rightarrow T(F)$ in $C\left(\overline{\mathbb{B}^{N \times m}}\right)$. The other terms are handled similarly, and so we get the desired inequalities.

Now we can prove our semicontinuity result, where we also allow for $x$-dependence of the integrand. The result could also be given without a boundary term, but its inclusion simplifies our proof. In the case $\mu=\mathcal{L}^{n}$, an analogous result was given in [14, Theorem 10].

Theorem 4.4. Let $\Omega \subset \mathbb{R}^{n}$ be a bounded Lipschitz domain with inner boundary normal $\nu_{\Omega}$, let $\mu \in \mathcal{M}^{+}(\Omega)$ with $\mathcal{L}^{n} \ll \mu$, and let $F \in \mathbf{R}\left(\Omega ; \mathbb{R}^{N \times n}\right)$ be nonnegative and $\mu \times \mathcal{B}\left(\mathbb{R}^{N \times n}\right)$-measurable such that $A \mapsto F(x, A)$ is quasiconvex for each fixed $x \in \bar{\Omega}$. Then the functional

$$
\begin{aligned}
\mathcal{F}(u):= & \int_{\Omega} F\left(x, \frac{d D u}{d \mu}\right) d \mu+\int_{\Omega} F^{\infty}\left(x, \frac{d D^{s, \mu} u}{d\left|D^{s, \mu} u\right|}\right) d\left|D^{s, \mu} u\right| \\
& +\int_{\partial \Omega} F^{\infty}\left(x, \frac{u}{|u|} \otimes \nu_{\Omega}\right)|u| d \mathcal{H}^{n-1}
\end{aligned}
$$

is weakly* sequentially lower semicontinuous in $\mathrm{BV}\left(\Omega ; \mathbb{R}^{N}\right)$.

Note that in the last term, $u$ is a boundary trace, see e.g. [5, Section 3.7]. Proof. Let $u_{j} \stackrel{*}{\rightarrow} u$ in $\operatorname{BV}\left(\Omega ; \mathbb{R}^{N}\right)$. Take a bounded Lipschitz domain $\Omega^{\prime} \ni \Omega$, and denote by $u_{j}^{e}, u^{e}$ the zero extensions of $u_{j}, u$ to $\Omega^{\prime} \backslash \Omega$. Since $\Omega$ is a bounded Lipschitz domain, we can use standard gluing theorems for BV functions, see e.g. [5, Proposition 3.21, Theorem 3.84, Theorem 3.86], to obtain that $u_{j}^{e} \in \operatorname{BV}\left(\Omega^{\prime} ; \mathbb{R}^{N}\right)$ with

$$
D u_{j}^{e}=\nabla u_{j} \mathcal{L}^{n}\left\llcorner\Omega+D^{s} u_{j}+u_{j} \otimes \nu_{\Omega} \mathcal{H}^{n-1}\llcorner\partial \Omega\right.
$$

and $\left\|u_{j}\right\|_{L^{1}\left(\partial \Omega ; \mathbb{R}^{N}\right)} \leq C\left\|u_{j}\right\|_{\mathrm{BV}\left(\Omega ; \mathbb{R}^{N}\right)}$ with $C$ depending only on $\Omega$; and similarly for $u^{e}$. By the weak* convergence, $u_{j}$ is a norm-bounded sequence in $\operatorname{BV}\left(\Omega ; \mathbb{R}^{N}\right)$, so we have that $u_{j}^{e}$ is a norm-bounded sequence in $\operatorname{BV}\left(\Omega^{\prime} ; \mathbb{R}^{N}\right)$ and that $u_{j}^{e} \rightarrow u^{e}$ in $L^{1}\left(\Omega^{\prime} ; \mathbb{R}^{N}\right)$. This implies that $u_{j}^{e} \stackrel{*}{\rightarrow} u^{e}$ in $\operatorname{BV}\left(\Omega^{\prime} ; \mathbb{R}^{N}\right)$. 
Since $F \in \mathbf{R}\left(\Omega, \mathbb{R}^{N \times n}\right), F^{\infty}(x, A)$ is continuous on $\bar{\Omega} \times \partial \mathbb{B}^{N \times n}$, which is a compact set. By the Tietze extension theorem, we can extend $F^{\infty}$ to $\overline{\Omega^{\prime}} \times \partial \mathbb{B}^{N \times n}$ as a continuous nonnegative function $\left(F^{e}\right)^{\infty}$. If we define $F^{e}(x, t A):=t\left(F^{e}\right)^{\infty}(x, A)$ for any $t \geq 0, A \in \mathbb{R}^{N \times n}$, and $x \in \overline{\Omega^{\prime}}$, we see that our notation is consistent in that the recession function of $F^{e}$ is indeed $\left(F^{e}\right)^{\infty}$. We also extend $\mu$ by $\mu^{e}:=\mu \mathrm{L} \Omega+\mathcal{L}^{N}\left\llcorner\left(\mathbb{R}^{n} \backslash \Omega\right)\right.$. Then we see that $F^{e} \in \mathbf{R}\left(\Omega^{\prime} ; \mathbb{R}^{N \times n}\right)$ is nonnegative and $\mu^{e} \times \mathcal{B}\left(\mathbb{R}^{N \times n}\right)$-measurable. We write

$$
\begin{aligned}
\mathcal{F}^{e}\left(u_{j}^{e}\right):= & \int_{\Omega^{\prime}} F^{e}\left(x, \frac{d D u_{j}^{e}}{d \mu^{e}}\right) d \mu^{e}+\int_{\Omega^{\prime}}\left(F^{e}\right)^{\infty}\left(x, \frac{d D^{s, \mu^{e}} u_{j}^{e}}{d\left|D^{s, \mu^{e}} u_{j}^{e}\right|}\right) d\left|D^{s, \mu^{e}} u_{j}^{e}\right| \\
= & \int_{\Omega} F\left(x, \frac{d D u_{j}}{d \mu}\right) d \mu+\int_{\Omega} F^{\infty}\left(x, \frac{d D^{s, \mu} u_{j}}{d\left|D^{s, \mu} u_{j}\right|}\right) d\left|D^{s, \mu} u_{j}\right| \\
& \quad+\int_{\partial \Omega} F^{\infty}\left(x, \frac{u_{j}}{\left|u_{j}\right|} \otimes \nu_{\Omega}\right)\left|u_{j}\right| d \mathcal{H}^{n-1} \\
= & \mathcal{F}\left(u_{j}\right),
\end{aligned}
$$

and similarly for $u^{e}$. We conclude that we need to prove that $\mathcal{F}^{e}\left(u^{e}\right) \leq$ $\liminf \operatorname{in}_{j \rightarrow \infty} \mathcal{F}^{e}\left(u_{j}^{e}\right)$. Pick first a subsequence (not relabeled) that gives this limit, and then by Theorem 2.3 and Corollary 2.4 we can pick a further subsequence (not relabeled) such that the sequence $D u_{j}^{e}$ generates a generalized Young measure $\nu=\left(\nu_{x}, \lambda_{\nu}, \nu_{x}^{\infty}\right)$, with respect to $\mu^{e}$. Clearly $\lambda_{\nu}\left(\partial \Omega^{\prime}\right)=0$, and then the barycenter of $\nu$ is $D u^{e}$, see the discussion after (2.7). Note that for any fixed $x \in \bar{\Omega}, F^{e}(x, \cdot) \in \mathbf{R}\left(\Omega, \mathbb{R}^{N \times n}\right) \cap \mathbf{Q}\left(\mathbb{R}^{N \times n}\right)$, so that we can apply Corollary 4.3 to obtain

$$
\begin{aligned}
& \liminf _{j \rightarrow \infty} \mathcal{F}^{e}\left(u_{j}^{e}\right)=\int_{\Omega^{\prime}}\left\langle F^{e}(x, \cdot), \nu_{x}\right\rangle d \mu^{e}+\int_{\Omega^{\prime}}\left\langle\left(F^{e}\right)^{\infty}(x, \cdot), \nu_{x}^{\infty}\right\rangle d \lambda_{\nu}(x) \\
& \quad \geq \int_{\Omega^{\prime}} F^{e}\left(x, \frac{d D u^{e}}{d \mu^{e}}\right) d \mu^{e}+\int_{\Omega^{\prime}}\left(F^{e}\right)^{\infty}\left(x, \frac{d D^{s, \mu^{e}} u^{e}}{d\left|D^{s, \mu^{e}} u^{e}\right|}\right) d\left|D^{s, \mu^{e}} u^{e}\right| \\
& \quad=\mathcal{F}^{e}\left(u^{e}\right) .
\end{aligned}
$$

\section{References}

[1] G. Alberti, Rank one property for derivatives of functions with bounded variation, Proc. Roy. Soc. Edinburgh Sect. A 123 (1993), no. 2, 239-274.

[2] J. J. Alibert and G. Bouchitté, Non-uniform integrability and generalized Young measures, J. Convex Anal. 4 (1997), no. 1, 129-147. 
[3] L. Ambrosio, G. Buttazzo, and I. Fonseca, Lower semicontinuity problems in Sobolev spaces with respect to a measure, J. Math. Pures Appl. (9) 75 (1996), no. 3, 211-224.

[4] L. Ambrosio and G. Dal Maso, On the relaxation in $\mathrm{BV}\left(\Omega ; \mathbb{R}^{m}\right)$ of quasi-convex integrals, J. Funct. Anal. 109 (1992), no. 1, 76-97.

[5] L. Ambrosio, N. Fusco, and D. Pallara, Functions of bounded variation and free discontinuity problems, Oxford Mathematical Monographs. The Clarendon Press, Oxford University Press, New York, 2000. xviii+434 pp.

[6] J. Ball, B. Kirchheim, and J. Kristensen, Regularity of quasiconvex envelopes, Calc. Var. Partial Differential Equations 11 (2000), no. 4, 333-359.

[7] B. Dacorogna, Direct methods in the calculus of variations, Second edition. Applied Mathematical Sciences, 78. Springer, New York, 2008. xii+619 pp.

[8] R. J. DiPerna and A. J. Majda, Oscillations and concentrations in weak solutions of the incompressible fluid equations, Comm. Math. Phys. 108 (1987), no. 4, 667-689.

[9] L. Evans and R. Gariepy, Measure theory and fine properties of functions, Studies in Advanced Mathematics. CRC Press, Boca Raton, FL, 1992. viii+268 pp.

[10] I. Fonseca and S. Müller, Relaxation of quasiconvex functionals in $\operatorname{BV}\left(\Omega, \mathbb{R}^{p}\right)$ for integrands $f(x, u, \nabla u)$, Arch. Rational Mech. Anal. 123 (1993), no. 1, 1-49.

[11] H. Hakkarainen, J. Kinnunen, P. Lahti, and P. Lehtelä, Relaxation and integral representation for functionals of linear growth on metric measures spaces, preprint 2014.

[12] B. Kirchheim and J. Kristensen, On Rank-One Convex Functions that are homogeneous of Degree One, preprint 2015.

[13] J. Kristensen, Lower semicontinuity in spaces of weakly differentiable functions, Math. Ann. 313 (1999), no. 4, 653710.

[14] J. Kristensen and F. Rindler, Characterization of generalized gradient Young measures generated by sequences in $W^{1,1}$ and BV, Arch. 
Ration. Mech. Anal. 197 (2010), no. 2, 539-598. Erratum, Ibid. 203 (2012), 693-700.

[15] J. Kristensen and F. Rindler, Relaxation of signed integral functionals in BV, Calc. Var. Partial Differential Equations 37 (2010), no. 1-2, 29-62.

[16] S. Müller, On quasiconvex functions which are homogeneous of degree 1, Indiana Univ. Math. J. 41 (1992), no. 1, 295-301.

[17] Y. G. Reshetnyak, The weak convergence of completely additive vector-valued set functions, Sibirsk. Mat. J. 9 1968, 1386-1394.

[18] F. Rindler, Lower semicontinuity and Young measures in BV without Alberti's rank-one theorem, Adv. Calc. Var. 5 2012, no. 2, $127-$ 159.

[19] W.P. Ziemer, Weakly differentiable functions. Sobolev spaces and functions of bounded variation Graduate Texts in Mathematics, 120. Springer-Verlag, New York, 1989. 